\title{
p-hub median problem for non-complete networks
}

\author{
İbrahim Akgün ${ }^{\mathrm{a}, *}$, Barbaros Ç Tansel ${ }^{\mathrm{b}, 1}$ \\ a Faculty of Enginering, Department of Industrial Engineering, Abdullah Gul University, Kayseri 38080, Turkey, \\ ${ }^{\mathrm{b}}$ Department of Industrial Engineering, Bilkent University, Ankara, Turkey
}

\section{A R T I C L E I N F O}

\section{Article history:}

Received 3 November 2017

Revised 22 January 2018

Accepted 22 February 2018

Available online 7 March 2018

Keywords:

Hub location

Integer programming

P-hub median

Network design

Non-complete networks

Incomplete hub network

Triangle Inequality

\begin{abstract}
A B S T R A C T
Most hub location studies in the literature use a complete-network structure as an input in developing optimization models. This starting point is not necessarily from assuming that the underlying real-world network (e.g., physical network such as road and rail networks) on which the hub system will operate is complete. It is implicitly or explicitly assumed that a complete-network structure is constructed from the shortest-path lengths between origin-destination pairs on the underlying real-world network through a shortest-path algorithm. Thus, the network structure used as an input in most models is a complete network with the distances satisfying the triangle inequality. Even though this approach has gained acceptance, not using the real-world network and its associated data structure directly in the models may result in several computational and modeling disadvantages. More importantly, there are cases in which the shortest path is not preferred or the triangle inequality is not satisfied. In this regard, we take a new direction and define the $p$-hub median problem directly on non-complete networks that are representative of many real-world networks. The proposed problem setting and the modeling approach allow several basic assumptions about hub location problems to be relaxed and provides flexibility in modeling several characteristics of real-life hub networks. The proposed models do not require any specific cost and network structure and allow to use the real-world network and its asociated data structure directly. The models can be used with the complete networks as well. We also develop a heuristic based on the proposed modeling aproach and present computational studies.
\end{abstract}

(C) 2018 Elsevier Ltd. All rights reserved.

\section{Introduction}

Hub facilities exist in many telecommunication and transportation systems where commodities (data, packages, passengers, etc.) are sent between many origin-destination (OD) pairs. In such systems, instead of establishing direct links (i.e., fully interconnected network) and sending flows between all OD pairs directly, some or all commodities are sent through one or more hubs that act as sorting, switching, connecting, and consolidation points.

A generic hub location problem involves determining the locations of hub facilities and the assignment of service routes between OD pairs. Different types of hub location problems, e.g., $p$-hub median, $p$-hub center, and hub covering, have been defined and extensively studied in the literature. See, for instance, Campbell et al. (2001) and Alumur and Kara (2008) for a review. However, several researchers, e.g., Campbell and O'Kelly (2012) and Contreras (2015), emphasize that there is a need to go beyond the classical hub location problems and define new ones that better

\footnotetext{
* Corresponding author.

E-mail address: ibrahim.akgun@agu.edu.tr (İ. Akgün).

1 Deceased
}

represent the real-world hub systems. Specifically, Campbell and O'Kelly (2012) state that much more effort should be directed at better modeling of specific air, ground, or water transportation systems and operations and incorporating both more realistic transportation costs and service measures along with other relevant aspects into models rather than solving very large-scale idealized hub location problems.

The source of motivation for our study is this stated need. We aim to develop a problem setting and modeling approach that will allow some limiting assumptions underlying most hub location models to be relaxed and hence to add flexibility and realism in modeling several characteristics of real-life hub systems. In the paper, we focus on the $p$-hub median version of the problem but our approach can be adapted to other hub location problems as well.

For a detailed discussion about the assumptions of the hub location models, see, e.g., Campbell and O'Kelly (2012), Contreras (2015), and Campbell et al. (2015). In the following, we discuss some assumptions (properties) together with their direct and indirect consequences and then explain how we address them.

To start with, we define five types of networks: (1) Real-world network (RealN): The physical network, e.g., road and rail networks, 
on which the hub system will operate. (2) Modeled network (MN): The network used as an input in developing a model for the problem. MN is not necessarily the same as RealN but may be obtained from RealN through preprocessing. (3) Hub network (HN): The subnetwork of MN that consists of the hub nodes, non-hub nodes, and the arcs on the service routes between OD pairs. (4) Hub-level network (HLN): The subnetwork of HN consisting of the hub nodes and the arcs connecting them. (5) Access network (AN): The subnetwork of HN consisting of the hub nodes, non-hub nodes, and access arcs that connect non-hub origin and destination nodes to hub nodes.

Most models assume that MN is a complete network with arc distances satisfying the triangle inequality (Assumption 1). This assumption is not necessarily a result of RealN because RealN may not be complete as is the case in most real-life networks, e.g., rail and road networks (or even if RealN is complete, its distances may not satisfy the triangle inequality). In such cases, even though not generally stated in most studies, researchers make an implicit assumption that a complete $\mathrm{MN}$ is constructed from the shortest path lengths between OD pairs in RealN through an algorithm such as the Floyd Algorithm (Floyd, 1962) (and hence arc distances satisfy the triangle inequality). However, this approach and the assumption have some implications and disadvantages: (1) The model size gets large very quickly. In an $n$-node complete network, the number of arcs is $n(n-1) / 2$ while it is at most $3 n-6$ in a planar network (Nishizeki and Chiba, 1998). (2) It is difficult to handle some situations, e.g., when some arcs in RealN are appropriate for the passage of small vehicles but not for large vehicles that are used for inter-hub transport. (3) The routing information on RealN can only be obtained after post-processing the solution on $\mathrm{HN}$ because an arc in a complete $M N$ (and hence in $\mathrm{HN}$ ) may actually correspond to a shortest path and not necessarily a single arc in RealN. (4) It is more challenging to model arc capacities on RealN and the interactions between facility location and routing decisions. (5) The approach cannot be applied for cases in which (i) using the shortest path costs is not necessarily preferred or correct, e.g., communication networks, and (ii) expecting the triangle inequality to hold is not realistic, e.g., passenger airline, train, and bus fares. (6) Cost model cannot take into account the fact that cost factors and their effects on different arcs of RealN may be different. In general, a standard transportation rate per unit distance per unit flow in all arcs is used. However, the incurred costs change depending on several factors. For example, costs are different for large- and small-size vehicles on different types of roads with different speed limits, congestion levels, and tolls, e.g., Transportation Research Board (2013) and Ricardo-AEA (2014). ((7) Cases in which there are multiple arcs between two nodes in RealN with different costs and service levels cannot be modeled. As an example, there are three options for the drivers in Turkey to move from one side of the Gulf of Izmit to the other, which is on the route between Istanbul and the cities in the west of Turkey: (i) using toll ferry with a travel time (cost) of about $1 \mathrm{~h}(\$ 25)$, (ii) driving around the gulf and through city center with a travel time (cost) of about $2 \mathrm{~h}(\$ 10)$, and (iii) using the toll bridge with a travel time (cost) about 4-6 min. (\$30). (8) Some definitions (e.g., service level or network topology) may become vague for some cases. For example, if there are hop constraints (e.g., the number of arcs on a route) for a road network, it is not the actual number of arcs in RealN but the shortest-path arcs in MN considered with the current approach if additional data and constraints are not used.

The aforementioned issues clearly indicate that there are several advantages of eliminating Assumption 1 and using RealN and its associated data structure directly in $\mathrm{MN}$, which is what we do in this paper. Specifically, we directly define the $p$-hub median problem on non-complete networks that are representative of most RealNs. This allows us to develop models that do not require any specific cost and network structures. Currently, there are no such models; all models use a complete network structure as MN. Marin et al. (2006) modify some hub location models to make them usable when the triangle inequality is not satisfied. However, modified models are based on other limiting assumptions to be mentioned.

There are three movement types in a hub system: collection from the origin to a hub, transfer between hubs, and distribution from the last hub to the destination. Most studies assign constant cost rates (per unit distance per unit flow) $\chi, \alpha$, and $\delta$ for collection, transfer, and distribution on all arcs, respectively, with $\alpha<\chi$ and $\alpha<\delta$ to capture economies of scale. In general, $\chi=\delta=1$ and $0 \leq \alpha \leq 1$. Thus, transportation costs on all inter-hub arcs are discounted by a constant factor of $\alpha$ independent of the actual amount of flow on the arcs (Assumption 2). Most models also assume that all flows are routed via a set of hubs (no direct flows between non-hub nodes) (Assumption 3). These three assumptions without any other restrictions imply that transportation cost is minimized when flows between OD pairs visit at most two hubs in a HN (Property 1), i.e., a route between an OD pair in an HN consists of at most three arcs, namely, collection (access), transfer (hub), and distribution (access) arcs. Given non-zero flows between all OD pairs, Property 1 in turn implies that all hubs are fully interconnected by the hub arcs, i.e., HLN is a complete network (Property 2).

Most studies including Marin et al. (2006) impose these two properties as topological requirements on HN. However, there may be cases where these properties are restrictive. For example, when the distances in MN do not satify the triangle inequality, there may be more than two hubs in the optimal routes. Similarly, if the setup costs for the hub arcs are significant, the complete-HLN topology may not be appropriate. Several studies (e.g., Campbell et al., 2005; Alumur et al., 2009; Calik et al., 2009; Yaman, 2009; Contreras et al., 2010; Campbell, 2010; Martins de Sá et al., 2013; Martins de Sá et al., 2015a, 2015b) have tried to address these shortcomings by allowing different HLN or AN topologies. However, they are restrictive as well due to Assumption 1, e.g., a tree-like HLN may not be constructed correctly when the triangle inequality is not satisfied.

Another issue related to Assumption 2 and Property 2 is about the modeling of economies of scale. The costs on all hub arcs are discounted by a factor of $\alpha$ because it is assumed that the flows are concentrated on the hub arcs. However, an analysis of the optimal flows indicates that some access arcs carry much higher flows than some hub arcs, but the costs on the access arcs are not discounted in the assumed cost structure. To remedy this weakness, some researchers (e.g., O'Kelly and Bryan [O'Kelly and Bryan, 1998]) develop models with flow-dependent discounts on the hub arc costs. Campbell et al. (2005a, 2005b) locate the hub arcs with each end point being a hub that may or may not be connected and thus relax Property 2. Campbell (2013) analyzes the optimal flows on the hub and access arcs by using different models with different data sets. He finds out that all models allow the access arc flows to exceed the hub arc flows at varying degrees and concludes that the requirement of Property 2 and the lack of discounts on the access arcs create the poor modeling of economies of scale rather than the form of cost discounting (flow-independent or flow-dependent) used for hub arc flows.

With these results in mind, we do not impose any HLN and AN topologies in our modeling approach. Costs on all hub arcs need not necessarily be discounted and costs on the access arcs may be discounted if necessary; it is possible to assign different discount rates for each type of movement (collection, transfer, and distribution) on each arc in RealN. We build our basic model with flowindependent costs on the hub and access arcs but show how to extend it to handle flow-dependent costs. The proposed approach is 


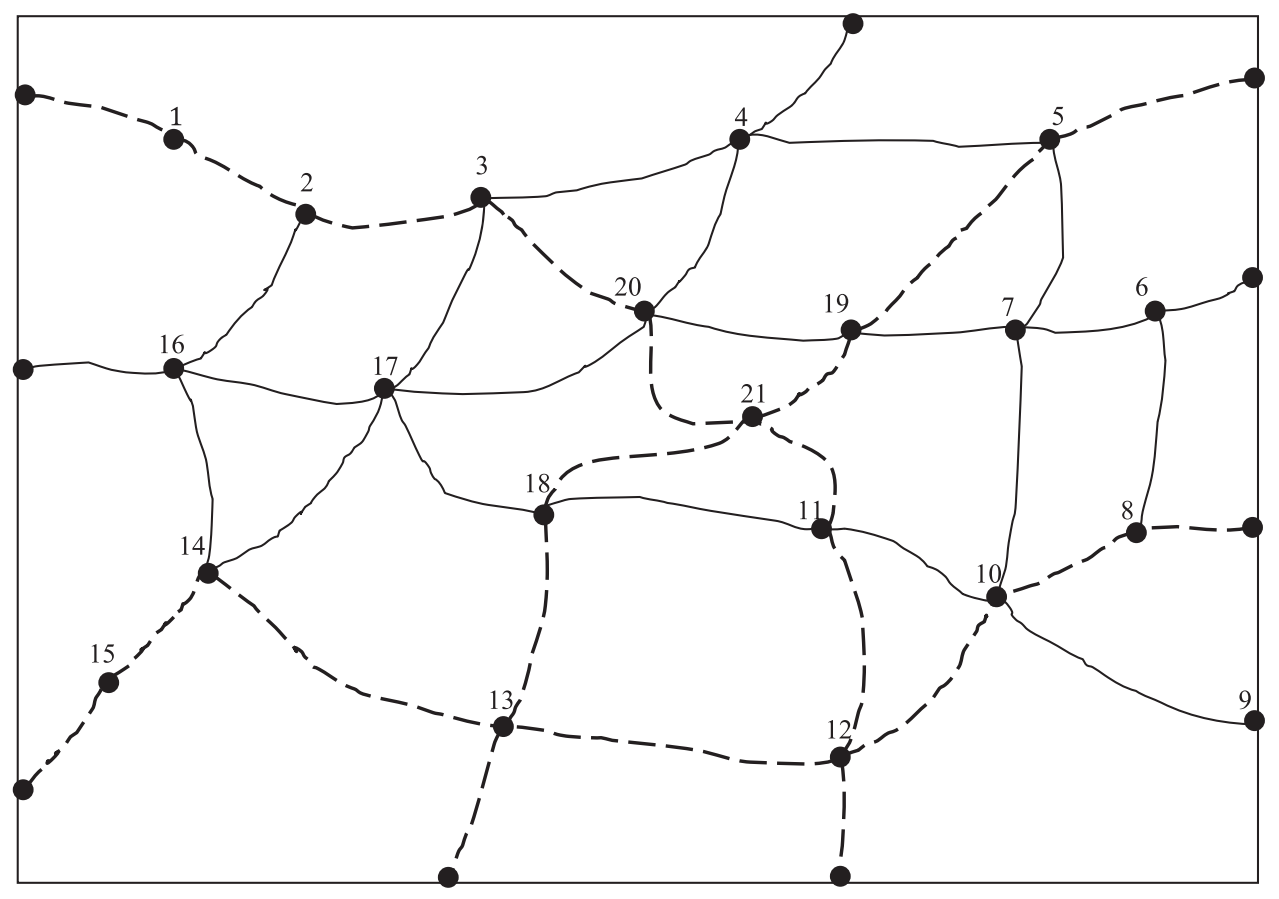

Fig. 1. An example network (RealN).

also flexible to address several needs that may arise, e.g., direct flows between non-hub nodes and tree-like HLN.

The rest of the paper is organized as follows. Section 2 defines the generalized $p$-hub median problem tailored to noncomplete networks. Section 3 gives the proposed models and Section 4 discusses the modeling features of the proposed approach. Section 5 presents a heuristic and gives computational results for the proposed models. Section 6 compares the new models with some previous models computationally and Section 7 concludes the paper.

\section{Problem definition}

Consider an undirected and connected network $G=(N, E)$ (RealN) with node set $N=\{1, \ldots, n\}$ and edge set $E$. A subset $S \subseteq N(D \subseteq N)$ is distinguished as the set of supply/origin (demand/destination) nodes. A node $i \in S$ generates flows $w_{i j}>0$ for some $j \in D$. For nodes $i$ and $j$ that are both in $S$ and $D$, it is not necessary that $w_{i j}=w_{j i}$.

Let $E^{*} \subseteq E$ be the set of edges appropriate for being hub arcs (e.g., roads (waterways) that allow the passage of over-size vehicles (large vessels)) and $N^{*} \subseteq N$ be the set of nodes incident to at least one edge in $E^{*}$. Then, $H$ is the set of potential hubs with $H=N^{*}$ or $H \subset N^{*}$ if other physical, administrative, or legal considerations lead to the elimination of certain nodes in $N^{*}$ and $G^{*}=\left(N^{*}, E^{*}\right)$ is the subnetwork of $G$ available for inter-hub transportation. Fig. 1 shows a sample network (RealN) where dashed lines and numbered points represent the edges in $E^{*}$ and nodes in $N$, respectively. The set of potential hub nodes is $\{1,2,3,20,21,11,12\} \cup\{5,19,21$, $18,13\} \cup\{15,14,13,12,10,8\}$ assuming that $H=N^{*}$.

Let $l_{i j}$ be the length of edge $\{i, j\}$. We define $\chi_{i j}, \alpha_{i j}$, and $\delta_{i j}$ as the cost of moving one unit of flow per unit length along the edge $\{i, j\}$ for collection, transfer, and distribution, respectively, with $\alpha_{i j}$ $\leq \chi_{i j}$ and $\alpha_{i j} \leq \delta_{i j}$ for an edge $\{i, j\}$ to capture economies of scale for inter-hub transport. All factors, including discounts, that affect the costs for an edge $\{i, j\}$ are assumed to be included in the computation of $\chi_{i j}, \alpha_{i j}$, and $\delta_{i j}$. Note that this definition of costs allows not only bridge arcs (an arc that connects two hubs but without the reduced unit flow cost of a transfer arc (Campbell et al., 2005a, 2005b) but also arcs with different cost rates.

The problem is then to choose $p$ nodes from a predefined set $H \subseteq N^{*}$ and find routes from nodes in $S$ to nodes in $D$ that visit at least one node in the selected hub set so that the total transportation cost is minimized. We assume a multi-assignment policy, i.e., each origin (destination) node can be allocated to more than one hub to send (receive) flows, and call the resulting problem as the Generalized Uncapacitated Multiple-Allocation p-Hub Median Problem (G-UMApHMP).

A particular case of G-UMApHMP is the well-known Uncapacitated Multiple-Allocation $p$-Hub Median Problem (UMApHMP) (e.g., Campbell, 1992; Skorin-Kapov et al., 1996; Ernst and Krishnamoorthy, 1998; Marin et al., 2006), where Assumptions (1)-(3) together with the resulting properties hold.

\section{Modeling generalized uncapacitated multiple allocation p-hub median problem}

In this section, we develop the proposed model for GUMApHMP, show how to reduce the size of original model, and indicate how to simplify the formulation for complete networks satisfying the triangle inequality.

\subsection{Development of the proposed model}

We model G-UMApHMP as a multi-commodity flow problem with side constraints on a three-layer network where first, second (middle), and third layers represent collection, transfer, and distribution components, respectively. Let $G=(N, A)$ be the directed version of $G=(N, E)$ where each $\{i, j\} \in E$ is replaced by a pair of directed $\operatorname{arcs}(i, j)$ and $(j, i)$ with $l_{i j}=l_{j i}$. We create two copies of $G=(N, A), G_{1}=\left(N_{1}, A_{1}\right)$ and $G_{3}=\left(N_{3}, A_{3}\right)$ with $N_{1}=\{11,12, \ldots, 1 n\}$, $N_{3}=\{31,32, \ldots, 3 n\}, A_{1}=\{(1 i, 1 j):(i, j) \in A\}$, and $A_{3}=\{(3 i, 3 j):(i$, $j) \in A\}$, to represent the first and third layers, respectively. The middle layer $G_{2}=\left(N_{2}, A_{2}\right)$ is defined by the edges $E^{*}$ with $N_{2}=N^{*}$ and $A_{2}=A^{*}$ where $A^{*}=\left\{(i, j) \in A:\{i, j\} \in E^{*}\right\}$. $\left(G_{2}\right.$ is not necessarily connected.) The three layers $G_{1}, G_{2}$, and $G_{3}$ are connected 

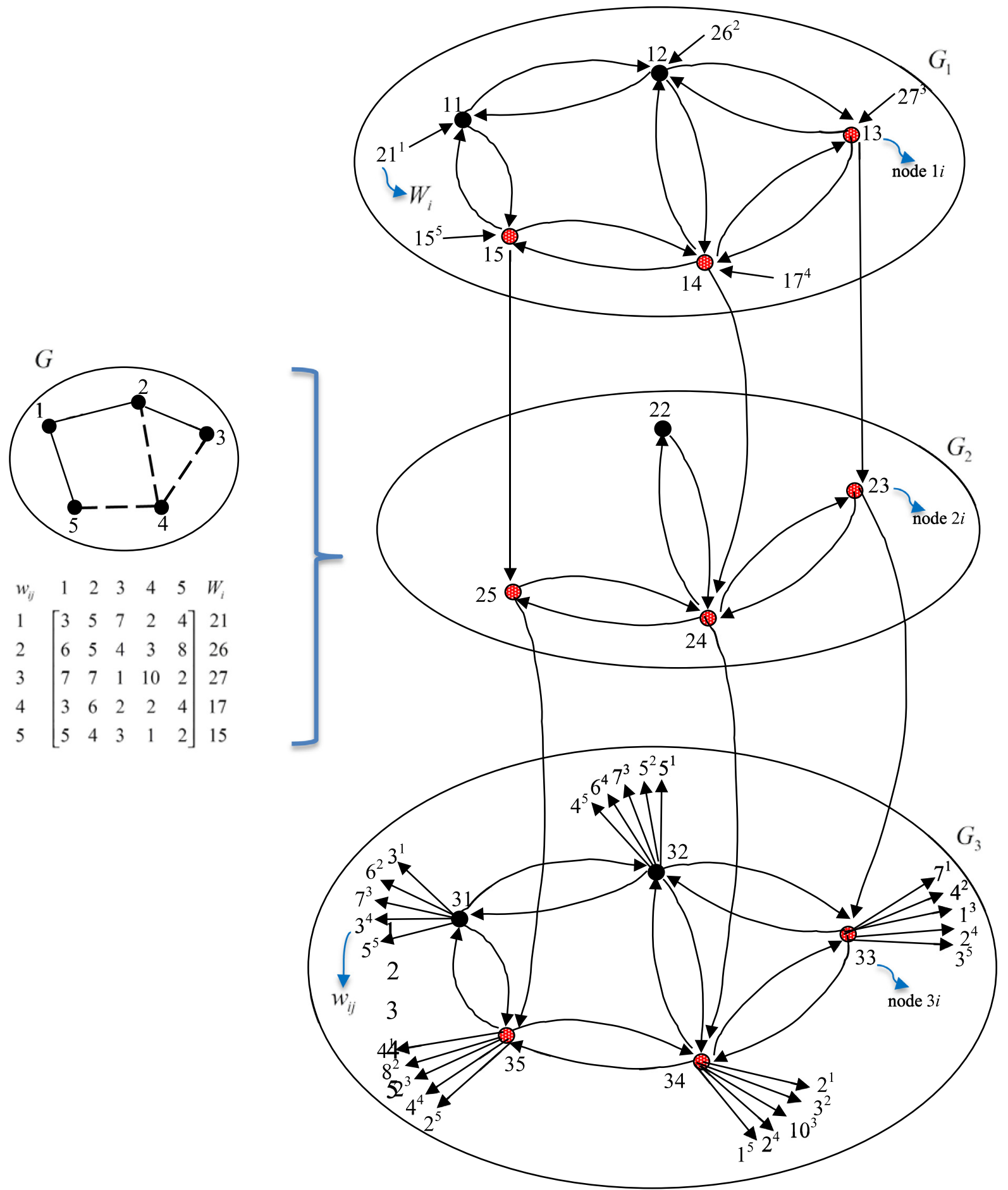

Fig. 2. Three-layer $M N G_{0}$ constructed from RealN $G$ where $E^{*}=\{\{4,5\},\{3,4\},\{2,4\}\}, N^{*}=\{2,3,4,5\}, H=\{3,4,5\}$, and $S=D=\{1,2,3,4,5\}$.

to each other by arcs of the form $(1 i, 2 i)$ and $(2 i, 3 i)$ for every $i \in H$. Let $A_{12}=\{(1 i, 2 i): i \in H\}$ and $A_{23}=\{(2 i, 3 i): i \in H\}$. Then, the final network is $G_{0}=\left(N_{0}, A_{0}\right)$ where $N_{0}=N_{1} \cup N_{2} \cup N_{3}$ and $A_{0}=A_{1} \cup A_{2} \cup A_{3} \cup A_{12} \cup A_{23}$. Note that $G_{0}=\left(N_{0}, A_{0}\right)$ is the MN and uses the structure of RealN.

Supply and demand nodes are in $G_{1}=\left(N_{1}, A_{1}\right)$ and $G_{3}=\left(N_{3}, A_{3}\right)$, respectively. A node $i$ in both $S$ and $D$ is a supply node $1 i$ in $G_{1}$ and a demand node $3 i$ in $G_{3}$. We define a different commodity for each $i \in S$. Let $w_{i j}$ be the demand of commodity $i$ at node $3 j \in N_{3}$ for $j \in D_{i}$ with $D_{i}=\left\{j \in D: w_{i j}>0\right\}, \quad i \in S$. Then, $W_{i}=\sum_{j \in D_{i}} w_{i j}$ is the total outbound flow at supply node $1 i \in N_{1}$ for $i \in S$. Fig. 2 shows a three-layer network (MN) constructed from RealN $G$ with $E^{*}, N^{*}, H, S, D, W_{i}$, and $w_{i j}$ as shown.

The three-layer network-structure idea has been used by Campbell et al. (2015, 2005b) as well. However, how the threelayer network structure is used and constructed in this paper is different from that in Campbell et al. (2015, 2005b). In Campbell et al. $(2015,2005 b), M N$ is a complete network satisfying Assumption 1 and hence the formulations are not dependent on the three-level network representation. However, the embedded network flow structure in the formulations is illustrated using a three-layer network. In the three-layer network in Campbell et al. 
(2015, 2005b), the top and bottom layers correspond to the destination and origin nodes, respectively, and the middle layer corresponds to the hub nodes. The collection (distribution) arcs are directed from the bottom (middle) layer to the middle (top) layer while transfer arcs connect hub nodes in the middle layer. In this paper, all flows occur in the layers and the flows between the layers are allowed only through vertical arcs (with zero costs) connecting hub nodes in different layers.

The cost of moving one unit of flow on an $\operatorname{arc} a, c_{a}$, is defined as follows $\left(\chi_{i j}=\chi_{j i}, \alpha_{i j}=\alpha_{j i}\right.$, and $\delta_{i j}=\delta_{j i}$ are assumed but not necessary.):

$c_{a}= \begin{cases}\chi_{i j} \times l_{i j} & \text { if } a=(1 i, 1 j),(i, j) \in A \\ \alpha_{i j} \times l_{i j} & \text { if } a=(2 i, 2 j),(i, j) \in A^{*} \\ \delta_{i j} \times l_{i j} & \text { if } a=(3 i, 3 j),(i, j) \in A \\ 0 & \text { if } a=(1 i, 2 i) \text { or } a=(2 i, 3 i), i \in H\end{cases}$

Let $x_{a k}$ be a flow variable for each arc $a \in A_{0}$ and each commodity $k \in S$. Define $y_{2 i} \in\{0,1\}, 2 i \in N_{2}$, for each $i \in H$ with $y_{2 i}=1$ if node $i$ is a hub and $y_{2 i}=0$ otherwise. The flow-based formulation has a flow conservation equation for each node in $N_{0}$ and each commodity $k$. We choose $p$ hubs via $\sum_{i \in H} y_{2 i}=p$. We allow the passage of flows from one layer to the other through vertical arcs (those in $A_{12}$ and $A_{23}$ ) only if their end nodes are selected as hubs.

Let $\beta$ be any node of $N_{0}$. With abuse of notation, if $\beta=1 i(\beta=3 i)$, i.e., in the collection (distribution) layer, we write $\beta \in N_{1}\left(\beta \in N_{3}\right)$. If $i \in S(i \in D)$, we write $\beta \in S(\beta \in D)$. Similarly, if $\beta=2 i$, i.e., in the transfer layer, we write $\beta \in N_{2}\left(=N^{*}\right)$. If $i \in H$, then we write $\beta \in H$. The requirement $b_{\beta k}$ at node $\beta$ for commodity $k$ is defined as $W_{\beta}=\sum_{j \in D_{i}} w_{i j}$ for $\beta=1 i, i \in S$, and as $-w_{k \beta}=-w_{k j}$ for $\beta=3 j, j \in D$. For all other nodes and $k \in S$, $b_{\beta k}=0$. Let $F_{\beta}^{\text {out }}$ be the forward star of a node $\beta \in N_{0}$ consisting of arcs whose tails are $\beta$ and $F_{\beta}^{\text {in }}$ be the inward star of node $\beta$ consisting of arcs whose heads are $\beta$.

With these definitions, the proposed model is given below.

Model MCFP: Multicommodity Flow p-Hub Median

\section{Formulation}

$z^{*}=\min _{x, y} \sum_{k \in S} \sum_{a \in A_{0}} c_{a} x_{a k}$

s.t. $\quad \sum_{a \in F_{\beta}^{\text {out }}} x_{a k}-\sum_{a \in F_{\beta}^{\text {in }}} x_{a k}=b_{\beta k} \quad \beta \in\left(N_{1} \cup N_{2} \cup N_{3}\right), \quad k \in S$

$$
\begin{gathered}
\sum_{i \in H} y_{2 i}=p \\
x_{(1 i, 2 i) k} \leq W_{k} y_{2 i} \quad i \in H, k \in S \\
x_{(2 i, 3 i) k} \leq W_{k} y_{2 i} \quad i \in H, k \in S \\
x_{a k} \geq 0 \quad a \in A_{0}, k \in S \\
y_{2 i} \in\{0,1\} \quad i \in H
\end{gathered}
$$

Objective function (1) minimizes the total transportation cost. Constraints (2) are flow-balance constraints for all nodes in the three-layer network and all commodities. Constraint (3) requires that $p$ hub nodes be selected. Constraints (4) and (5) allow the passage of flows from the first to the second layer and from the second to the third layer only through vertical arcs whose heads and tails are hubs, respectively. Constraints (6) and (7) define the decision variables.

\subsection{Reduction of the size of the proposed model MCFP}

Because there are no capacity constraints in MCFP, the leastcost paths (LCPs) must be used between OD pairs in the optimal solution. So, by defining $x_{a k}$ only for the arcs on LCPs that each commodity can use in each layer, the size of MCFP can be reduced significantly.

We need the arcs on LCPs (1) from $1 k, k \in S$, to $1 i, \quad i \in H$, in $G_{1}$, (2) from $2 i, \quad i \in H$, to $2 j, \quad j \in H$, with $2 i \neq 2 j$, in $G_{2}$, and (3) from $3 i, \quad i \in H$, to $3 j, j \in D$, in $G_{3}$. We determine LCPs by solving a set of single-commodity flow models (SCMs). In each layer, the number of SCMs solved is equal to the number of origin nodes. In each SCM, each origin node has a supply equal to the cardinality of the destination nodes and each destination node has a demand of 1 . In $G_{1}$, each SCM is solved for a different commodity $k \in S$. In $G_{2}$ and $G_{3}$, each SCM is solved for a specific commodity $k$ and all other commodities $k^{\prime} \neq k$ are allowed to use the same paths.

Let $A_{k}^{1}, A_{k}^{2}$, and $A_{k}^{3}$ denote the set of directed arcs with positive flows in the solution of a SCM for commodity $k$ in $G_{1}, G_{2}$, and $G_{3}$, respectively. Then, we define $x_{a k}$ only for $a \in \bar{A}_{k}$ where $\bar{A}_{k}=A_{k}^{1} \cup A_{k}^{2} \cup A_{k}^{3} \cup A_{12} \cup A_{23}$. The sizes of MCFP and the reduced model RMCFP are compared in Appendix A.

\section{Model RMCFP: Reduced Multicommodity Flow P-Hub}

\section{Median Formulation}

In addition to constraints (3)-(5) and (7)

$z^{*}=\min _{x, y} \sum_{k \in S} \sum_{a \in \bar{A}_{k}} c_{a} x_{a}$

$$
\begin{aligned}
& \text { s.t. } \sum_{a \in\left(F_{\beta}^{\text {out } \left.\cap \bar{A}_{k}\right)}\right.} x_{a k}-\sum_{a \in\left(F_{\beta}^{\text {in }} \cap \bar{A}_{k}\right)} x_{a k}=b_{\beta k} \\
& \beta \in\left(N_{1} \cup N_{2} \cup N_{3}\right), k \in S \\
& x_{a k} \geq 0 \quad a \in \bar{A}_{k}, k \in S
\end{aligned}
$$

\subsection{Simplification of MCFP for complete networks satisfying the} triangle inequality

MCFP can solve problems defined on complete networks as given. However, if the costs (obtained by multiplying arc distances with unit flow arc costs) of the network MN associated with a layer satisfy the triangle inequality, simplifications can be made in the constraints of MCFP associated with that layer. (1) In $G_{1}, x_{a k}$ are defined for $a=(1 k, 1 i)$ and flow balance constraints are expressed for nodes $1 k$ and $1 i$ where $k \in S$ and $i \in H$. (2) In $G_{2}$, flow balance constraints are defined only for nodes $2 i$ with $i \in H$. (3) In $G_{3}$, flow balance constraints are expressed for $3 i$ with $i \in$ $(H \cup D)$. If a node is both a demand node and a hub node, flowbalance constraints are adapted accordingly. The resulting formulation with all simplifications applied to all levels is referred to as CMCFP (MCFP for Complete Networks Satisfying the Triangle Inequality). Unless specifically stated, MCFP will be used to refer to all three models.

Note that even if the arc distances satisfy the triangle inequality, the costs for each layer may violate the triangle inequality because different unit flow arc costs may apply for the arcs in each layer. This is why we require the costs to satisfy the triangle inequality in each layer.

\section{Features of the proposed modeling approach}

We use simple problems/cases to explore the advantages and features of the proposed modeling approach. Unless stated otherwise, $E^{*}, N^{*}, H, \quad w_{i j}, \alpha_{i j}$ and $z^{*}$ in the problems are as given in the figures. For all cases, $\chi_{i j}=\delta_{i j}=1$ and $p=3$. 

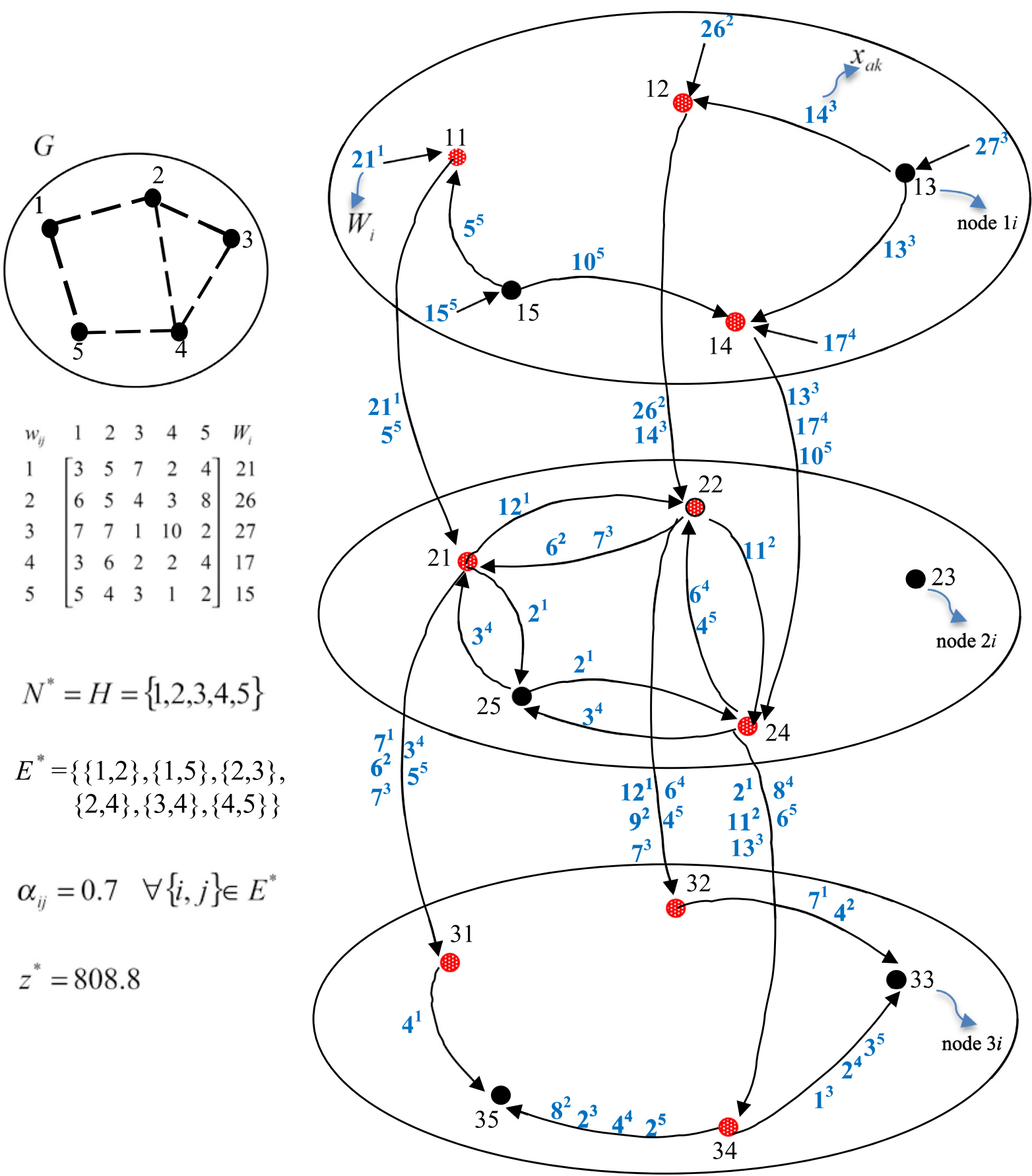

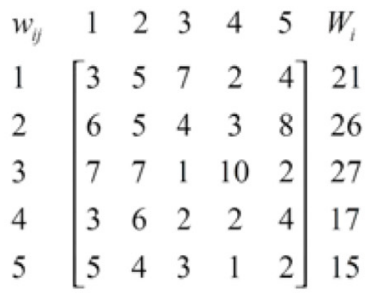

$N^{*}=H=\{1,2,3,4,5\}$

$E^{*}=\{\{1,2\},\{1,5\},\{2,3\}$, $\{2,4\},\{3,4\},\{4,5\}\}$

$\alpha_{i j}=0.7 \quad \forall\{i, j\} \in E^{*}$

$z^{*}=808.8$

Fig. 3. Optimal solution when all edges are appropriate for inter-hub movements.

The routing information on RealN $G$ can be obtained from the solution of MCFP. Consider the solution in Fig. 3 with all edges in $G$ being appropriate for inter-hub movement. The selected hubs are 1 , 2 , and 4 . The route from 11 to 34 on $\mathrm{HN}$ is $11-21-25-24-34$, which corresponds to the route $1-5-4$ on $G$. Non-hub node 5 is on the route from hub 1 to hub 4 because 5 is on the min-cost path from 1 to 4 on $G$, which is not possible to observe when Assumption 1 holds. If we were to use the complete network structure as $M N$, the route from 1 to 4 on MN would be 1-4 and HLN would be complete. In the following, we will refer to Fig. 3 several times to show how the solutions change in different cases.

In MCFP, HLN can be incomplete. HLN in Fig. 3 can be viewed as complete in the sense that all hubs are connected. Now consider Fig. 4 where $E^{*}$ in Fig. 3 is restricted as given. With this modifica- tion, the selected hubs are the same as in Fig. 3; however, HLN is not complete (no flow between 21 and 22). The flows on the arcs between 21 and 22 in Fig. 3 are rerouted as shown in Fig. 4 and $z^{*}$ increases from 808.8 to 868.8 .

In MCFP, HLN can be disconnected. Assume that $E^{*}=\{\{1,5\},\{2$, $4\},\{3,4\}\}$ in Fig. 3. In this case, the same hubs are selected. However, hub 21 is not connected to other hubs (22 and 24), i.e., hub 21 is an isolated hub, and only the arcs between 22 and 24 are transfer arcs. Hub 21 serves as a distribution center for itself and receives its demands from other sources in the first and third layers. $z^{*}$ increases from 808.8 to 888.3 .

MCFP allows to assign different arc cost rates to different types of movements in the network. Suppose that $\alpha_{i j}=0.7$ for $E^{*}=\{\{1$, $5\},\{2,4\},\{3,4\},\{4,5\}\}$ and $\alpha_{i j}=0.9$ for $E^{*}=\{\{1,2\},\{2,3\}\}$ in Fig. 3. 

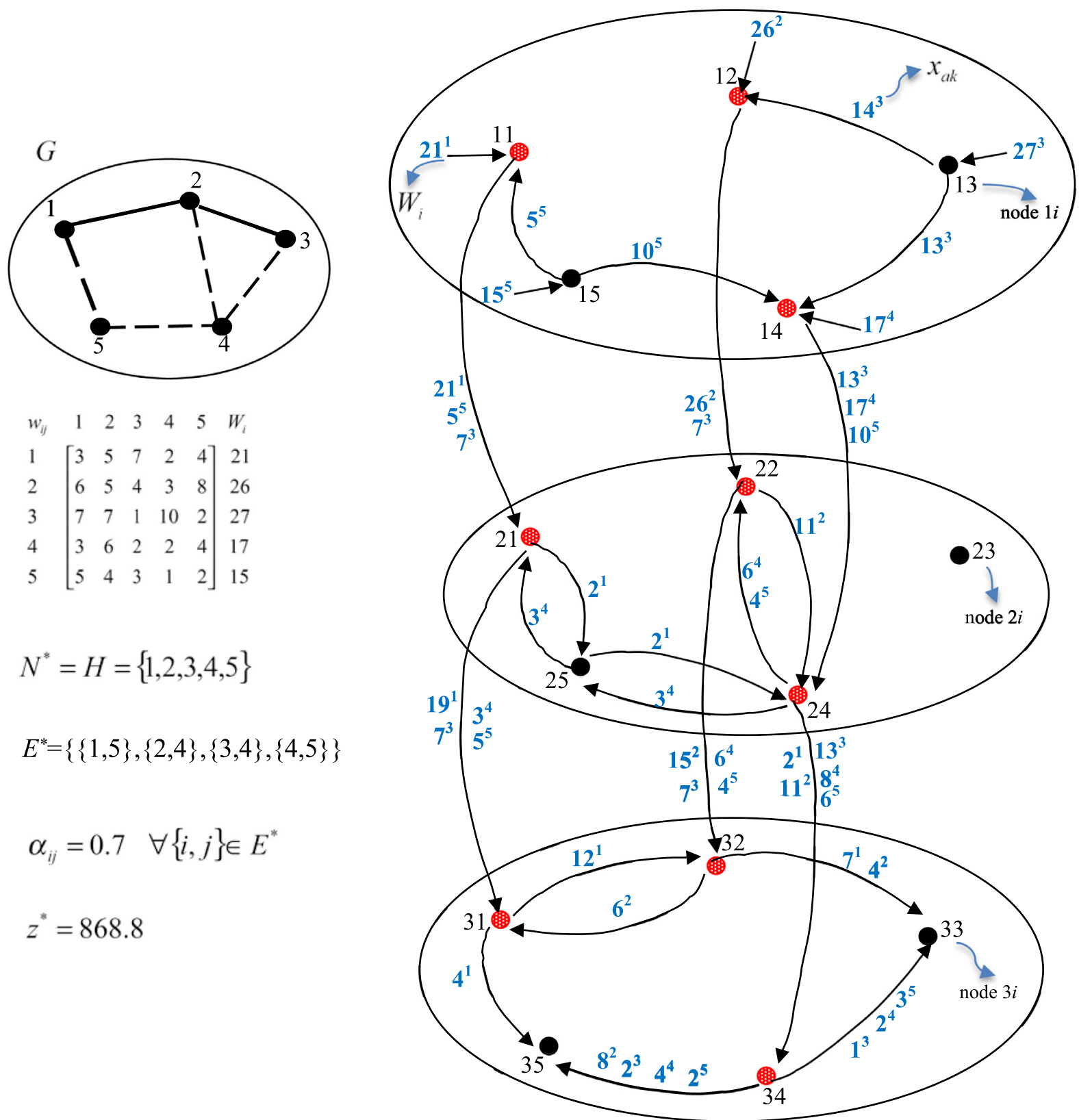

Fig. 4. Optimal solution when inter-hub movements are restricted to some edges.

In this case, the same solution in Fig. 3 is obtained but $z^{*}$ increases from 808.8 to 848.8 . Different cost rates may be set for $\chi_{i j}$ and $\delta_{i j}$ as well.

MCFP can be extended to handle multiple arcs between two nodes in RealN. In the case of Gulf of Izmit, three arcs with different cost rates are needed to represent the alternatives (i.e, ferry, bridge, and motorways) from one side of the gulf to the other. This can be addressed in MCFP by adding an index to represent arcs between two nodes. Alternative modes of transportation or different types of vehicles with a common mode can be modeled similarly.

In MCFP, node/arc capacities in RealN can be modeled easily. Suppose that the capacity between nodes 1 and 2 is 20 in $G$ in Fig. 3. Without restriction, the total amount of flow is 25 (between 21 and 22). To model the requirement, we can add the constraint $\sum_{i=1,2,3} \sum_{k \in S}\left(x_{(1 i, 2 i) k}+x_{(2 i, 1 i) k}\right) \leq 20$. In this case, the amount of flow between nodes 21 and 22 decreases to 20 . Five units of decrease is achieved by routing 5 units of commodity 3 through 13-
12-11-21-31 and $z^{*}$ increases from 808.8 to 820.8 . Additional capacity restrictions, e.g., different hub and access arc capacities, can be modeled similarly.

MCFP can be extended to handle cases where direct transportation between non-hub nodes is desired. To achieve this, we add direct arcs of the form $(1 i, 3 i)$ for $i \in N$. Consider Fig. 5 where $N^{*}=\{2,3,4,5\}$ and $H=\{2,3,4\}$ and $w_{i j}$ is the same as before except that $w_{15}=40$ and $w_{51}=50$. When the problem is solved, direct flows between nodes 1 and 5 are observed (on the dashed lines in Fig. 5). The movements between non-hub nodes occur in the first and/or third layers. In the example, additional costs for direct transportation are not assumed; however, if needed, they can be defined on $(1 i, 3 i)$ and incorporated into the objective function.

MCFP assumes flow independent costs on the arcs. However, it can be extended to handle flow-dependent costs. Here we show the extension assuming flow-dependent hub arc costs but flowdependent access arc costs may be incorporated similarly. Suppose 

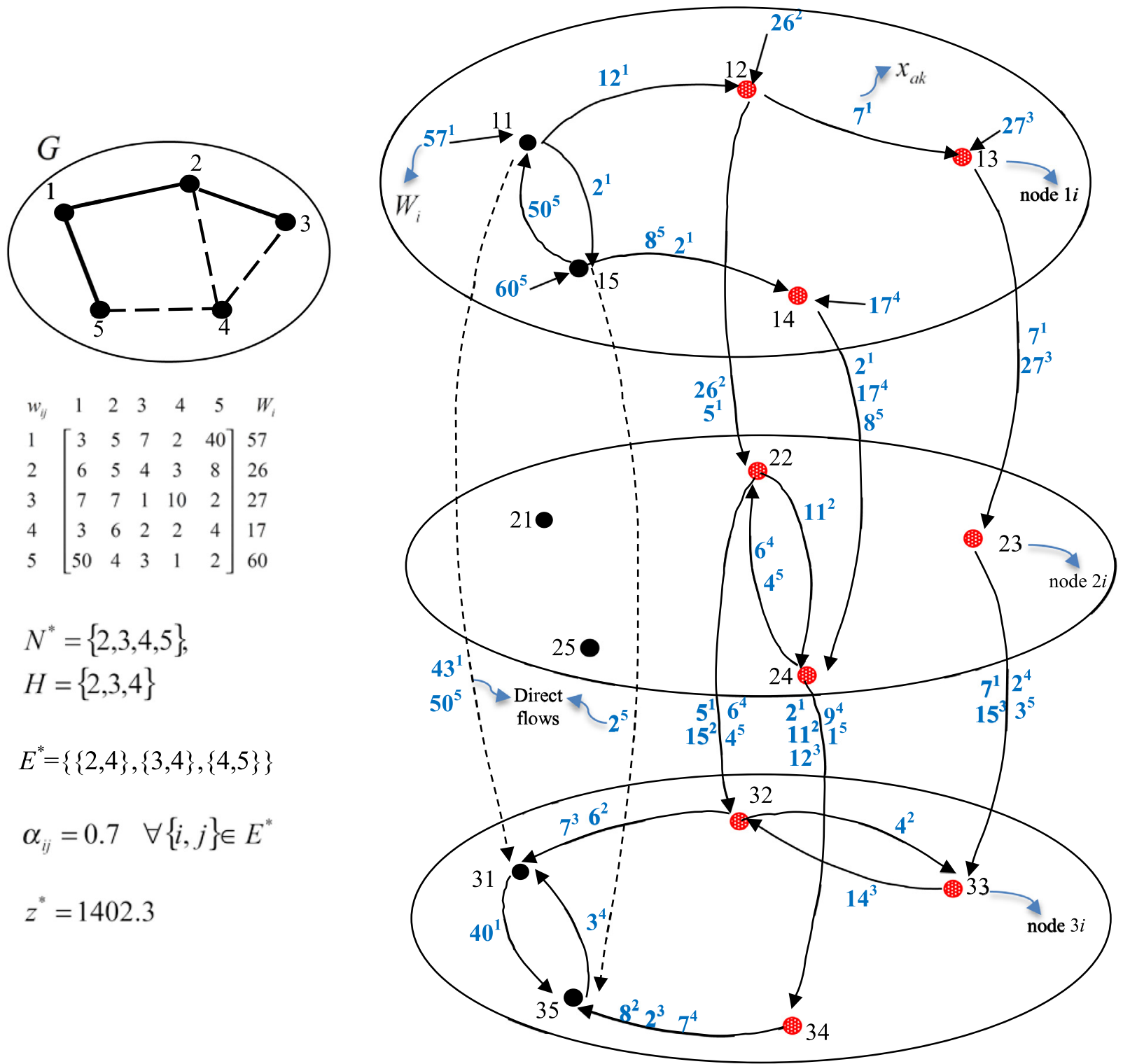

Fig. 5. Optimal solution when direct transportation is allowed. Dashed lines from the first to the third layer indicate direct transportation between associated nodes.

that a piecewise linear cost function with $q$ segments (or modes) characterizes the cost per unit length on an arc $a=(2 i, 2 j),(i, j) \in$ $A^{*}$. The slope of each line segment, $\alpha_{a q}$, gives the unit transportation cost per unit length on arc $a$ when mode $q$ is used, such that $\alpha_{a 1}>\alpha_{a 2}>\ldots>\alpha_{a q}$. Define $L_{a q}$ and $U_{a q}$ as the lower and upper bounds on the amount of flow on arc $a$ for mode $q$, respectively. To define the model, let (1) $\eta_{a q}$ be a binary variable that takes the value of 1 when mode $q$ is used on arc $a$ and 0 otherwise and (2) $R_{a q}$ be the total amount of flow on arc $a$ when mode $q$ is used.

Model FDMCFP: MCFP with Flow Dependent Hub Arc Costs

In addition to constraints (2)-(7)

$$
\begin{aligned}
& z^{*}=\min _{x, y, \eta, R} \sum_{k \in S} \sum_{a \in A_{1}} \chi_{a} l_{a} x_{a k}+\sum_{a \in A_{2}} \sum_{q} \alpha_{a q} l_{a} R_{a q}+\sum_{k \in S} \sum_{a \in A_{3}} \delta_{a} l_{a} x_{a k} \\
& \sum_{q} \eta_{a q} \leq 1 \quad a \in A_{2} \\
& \sum_{q} R_{a q}=\sum_{k \in S} x_{a k} \quad a \in A_{2} \\
& L_{a q} \eta_{a q} \leq R_{a q} \leq U_{a q} \eta_{a q} \quad a \in A_{2}, q
\end{aligned}
$$

$\eta_{a q} \in\{0,1\} \quad a \in A_{2}, q$

$R_{a q} \geq 0 \quad a \in A_{2}, q$

Constraints (12) require that at most one mode be selected. Constraints (13) compute the total flow on each arc. Constraints (14) set lower and upper bounds on the amount of flow on each arc for each mode. Constraints (15) and (16) define the decision variables. Objective function (11) minimizes the total cost. The first and third terms in (11) compute the total cost in the first and third layers, respectively. In the second term, the cost rate that will be active is determined depending on the selected mode, i.e., the total amount of flow on each arc. This formulation ignores fixed cost for using a mode but, if applicable, it can be incorporated by multiplying with $\eta_{a q}$. As an example, we modify the problem in Fig. 3 by defining $\alpha_{i j}$ with three modes as shown in Fig. 6. In the optimal solution, flow values on all transfer arcs are between 11-20, i.e., mode 2 is selected for all arcs. The total flow of 5 between $21-25$ and $24-25$ in Fig. 3 is not observed in Fig. 6. That is, the number of hub arcs with less flows than access arcs decreases (but is not 


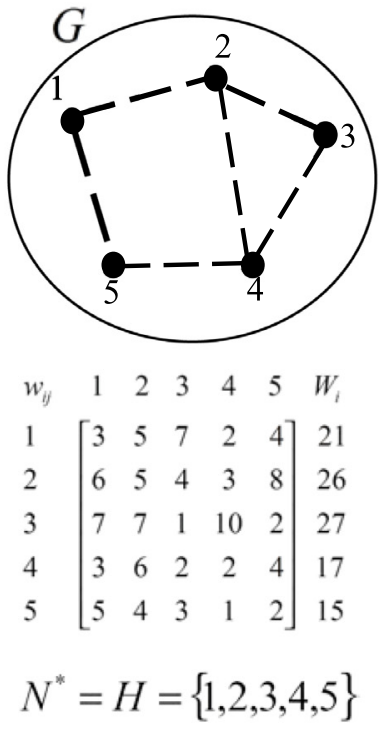

$E^{*}=\{\{1,2\},\{1,5\},\{2,3\}$, $\{2,4\},\{3,4\},\{4,5\}\}$

$\alpha_{i j}=\left\{\begin{array}{cc}1.0 & 0 \leq \text { flow } \leq 10 \\ 0.7 & 11 \leq \text { flow } \leq 20 \\ 0.6 & 21 \leq \text { flow } \leq 106\end{array}\right.$

$$
z^{*}=901.3
$$

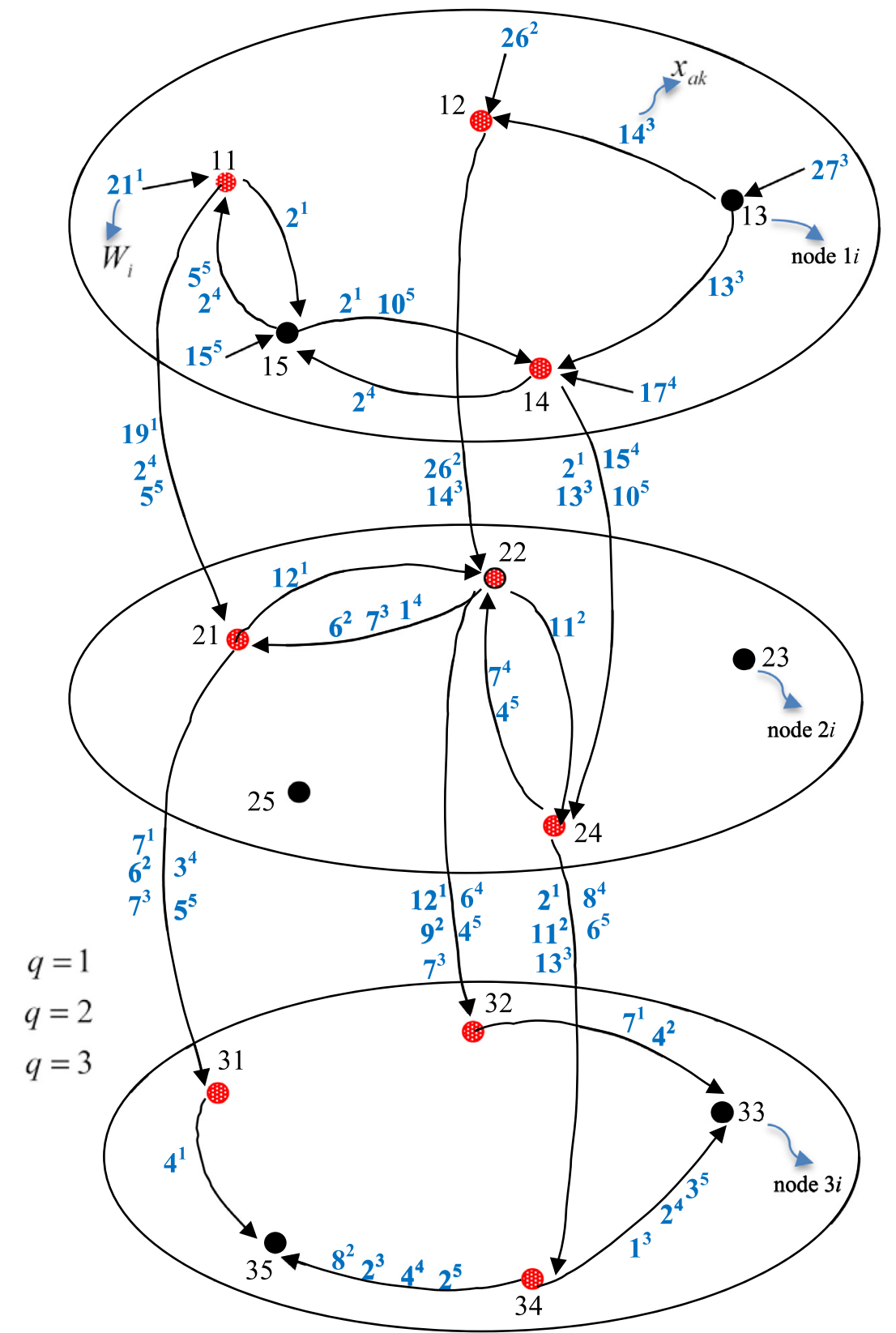

Fig. 6. Optimal solution when costs are flow-dependent eliminated) but the cost increases. When $\alpha_{i j}=0.5$ for $q=3$, mode 3 is selected for all hub arcs. When lower/upper bounds and discount rates are changed, different modes for different arcs are observed as well.

MCFP can also be cast as a Hub Arc Location Problem (HAL) where hub arcs rather than hub nodes are located (Campbell et al., 2005a, 2005b). The optimal solution to HAL may include two types of arcs in the transfer component: (1) hub arcs connecting two hubs with unit flow costs discounted by a factor of $\alpha$ and (2) bridge arcs connecting two hubs but without the discounted unit flow costs. We do not make this differentiation in MCFP because we already allow a different unit flow cost (hence discount factor) for each arc in the transfer layer and multiple arcs between two nodes (may be used for the case where hub arcs and bridge arcs are allowed to coincide). In HAL, a hub arc directly connects two hubs and hence each end node of a located hub arc is a hub. In MCFP, however, this is not required because we allow optimal paths that can traverse non-hub nodes in the HLN. For example, in Fig. 3, the route from 21 to 24 consists of hub arcs 21-25 and $25-24$ but node 25 is not a hub. It is possible to have a hub arc whose end nodes are not hubs as well. In this regard, we develop a formulation that treats all arcs with positive flows in the transfer layer as hub arcs. We remark that the located hub arcs in MCFP are not the shortest-path arcs but the arcs on RealN.

To formulate HAL, let $A_{(2 i, 2 j)}$ be a binary variable that takes on the value of 1 if hub arc $(2 i, 2 j)$ with $\{i, j\} \in E^{*}, \quad i<j$, is located and 0 otherwise. We define hub arcs as undirected but allow flows in both directions. Defining $r$ as the number of hub arcs to be located, we can state the formulation as follows.

\section{Model HALMCFP: MCFP with Hub Arc Location}

In addition to (1), (2), (4)-(7)

$\sum_{\{i, j\} \in E^{*}, i<j} A_{(2 i, 2 j)}=r$ 
$x_{(2 i, 2 j) k}+x_{(2 j, 2 i) k} \leq W_{k} A_{(2 i, 2 j)} \quad\{i, j\} \in E^{*}, i<j, k \in S$

$v A_{(2 i, 2 j)} \leq \sum_{k \in S} x_{(2 i, 2 j) k}+\sum_{k \in S} x_{(2 j, 2 i) k} \quad\{i, j\} \in E^{*}, i<j$

$y_{2 j} \leq \sum_{i \in N^{*}, i<j} A_{(2 i, 2 j)}+\sum_{i \in N^{*}, \quad} A_{(2 j, 2 i)} j \in H$

$A_{(2 i, 2 j)} \in\{0,1\} \quad\{i, j\} \in E^{*}, i<j$

Constraint (17) ensures that $r$ hub arcs will be located. Constraints (18) do not allow any flows on an arc that is not selected as a hub arc. Constraints (20) require that a hub arc be incident to a hub node. Constraints (19) prevent an arc from being assigned as a hub arc unless there are $v$ units of flow on the arc. This set of constraints is actually not a part of HAL (Campbell et al., 2005a, 2005b) and not required for HALMCFP to work correctly. We add this constraint because lower bounds on the amount of flow on the hub arcs may help decrease the number of access arcs that carry higher flows than some hub arcs and thereby allow better modeling of economies of scale.

Different HN (HLN) designs may be obtained depending on the values of $r$ and $v$ with different costs. If $r$ is set a very low value, cost may be high. On the other hand, if $r$ is set a very high value, many hubs may be located because of the minimization objective. As an example, for the problem in Fig. 3, if we solve HALMCFP with $v=1$ and $r=3, z^{*}=795$ and the resulting number of hubs is 5 , i.e., all nodes are hub nodes, which may not be desired. In this regard, it may be a good practice to restrict the number of hubs by adding constraint (3) in addition to the number of hub arcs in order to obtain acceptable HLN structures. Fig. 7 illustrates the optimal solution for a problem specifically designed to obtain a HLN consisting of non-hub nodes and hub arcs whose endpoints are not hubs. Due to the restrictions with respect to $H$ and $E^{*}, z^{*}$ increases to 1181.2 .

MCFP can be adapted to address single-assignment policy where all the incoming and outgoing traffic of each node is routed through a single hub. To formulate the problem, let $\tau_{1 k, 2 j}$ be a binary variable that takes on the value of 1 if node $1 k \in N_{1}$ is assigned to hub node $2 j \in N_{2}$ with $j \in H$ and 0 otherwise.

\section{Model SAMCFP: MCFP with Single Assignment}

In addtion to (1)-(7)

$\sum_{j \in H} \tau_{1 k, 2 j}=1 \quad k \in N$

$x_{(1 j, 2 j) k}=W_{k} \tau_{1 k, 2 j} \quad k \in S, j \in H$

$x_{(2 j, 3 j) l}=\sum_{k \in N} \sum_{m=3 k} w_{l m} \tau_{1 k, 2 j} \quad l \in S, j \in H$

$\tau_{1 k, 2 j} \in\{0,1\} \quad k \in N, j \in H$

Constraints (22) assign each node to a single hub node. Constraints (23) and (24) ensure that flows outgoing from and flows incoming to a node move through a single hub, respectively. Specifically, constraints (23) require that all supply of commodity $k$ move from the first to the second layer through hub node $2 j$ to which node $1 k$ is assigned. Constraints (24) necessitate that the amount of flow for a specific commodity $l$ moving from the second to the third layer through hub node $2 j$ be equal to the sum of demands of commodity $l$ at the nodes $3 k$ for nodes $1 k \in N_{1}$ that are assigned to hub node $2 j$. Fig. 8 gives the optimal solution for the problem in Fig. 3 with the single-assignment policy. The selected hub nodes are the same as in Fig. 3; however, each node gets service from a single hub. For example, node 3 gets service from hubs 2 and 4 in Fig. 3 while it gets service from node 4 in Fig. 8. Accordingly, $z^{*}$ increases to 972.4 .

In addition to the above, several other features can be modeled with MCFP. For example, fixed/variable costs for transportation or for construction of the arcs and nodes, lower/upper bounds on the number of nodes/arcs between two nodes (in the access and/or transfer layers), and requirements with regard to the HLN and AN topologies, e.g., tree structure, may be incorporated.

The previous discussion indicates that MCFP is flexible enough to model many different features existing in real-life hub systems. Some of these features are challenging, if not impossible, to model with the current models. However, there is a need to study these features separately and in detail for further analysis and insights.

\section{Solution of the proposed models and computational tests}

Our aim for this study is to analyze the solvability of MCFP and develop a solution methodology using standard optimization software. In this regard, we conduct computational tests using standard software and develop a heuristic based on MCFP.

It is also possible to develop more advanced solution algorithms, e.g., decomposition-based algorithms like the ones in Contreras et al. (2011), Camargo et al. (2008), and Camargo et al. (2009), because MCFP decomposes into easily-solvable problems. For example, when the variables $y_{2 i}$ in (4)-(5) are fixed, the formulation can be decomposed into single-commodity flow models appropriate for parallel programming. However, we leave the exploration of such properties for further research.

\subsection{Proposed heuristic RFHEUR}

RFHEUR is essentially composed of two steps. The first step determines a candidate hub set $H_{C}$ and the second step finds a feasible solution by selecting $p$ hub locations from $H_{C}$.

Let RMCFP_Rel be a relaxation of RMCFP obtained by removing the flow-balance constraints (9), $\beta \in N_{3}$, in RMCFP. To determine $H_{C}$, we solve RMCFP_Rel for $k$ times, each time with a different hub set $\bar{H}_{k} \subseteq H$. Let $y_{2 i}^{k}$ and $H_{k}=\left\{i \in \bar{H}_{k}: y_{2 i}^{k}=1\right\}$ be the location decisions and the set of candidate locations obtained in the $k$ th solution of RMCFP_Rel, respectively. While solving RMCFP_Rel for the $k$ th time, the hub set is restricted to $\bar{H}_{k}=H-\bigcup_{\bar{k}<k} H_{\bar{k}}$, i.e., the sets of candidate locations found in the previous $(k-1)$ solutions are eliminated from the hub set $H$. Then, $H_{C}=\bigcup_{k} H_{k}$ and $\left|H_{C}\right|=p \times k$. In the second step of the heuristic, we solve RMCFP with a restricted hub set, i.e., $H=H_{C}$, to find a feasible solution for the problem.

RMCFP_Rel is a mixed integer program (MIP) but solved to optimality in a matter of seconds on a PC with $2.50 \mathrm{GHz}$ Intel Core i7-4710HQ processor and 16 GB of RAM using standard optimization software. RMCFP with the restricted hub set $H_{C}$ is also easy for many practical problems; however, it may be as difficult to solve as the original problem depending on $k$, i.e., the size of $H_{C}$, and $|N|$. When $k=1$, we can fix the facility location decisions in RMCFP and solve it as an LP in a short time. However, for $k \geq 2$, RMCFP with $H_{C}$ may be difficult to solve especially for large values of $k$ when the network size is large. Our computational studies indicate that good solutions can be obtained with $k=1,2$.

\subsection{Computational studies for the proposed models}

We conduct computational studies to observe the performance of (1) the models using standard optimization software and (2) the proposed heuristic. Computational tests are performed on two sets of problems, namely, TR81(C) problems and PMED problems. TR81 problems are defined on an 81-node non-complete network (RealN) derived from the highway transportation network in Turkey 

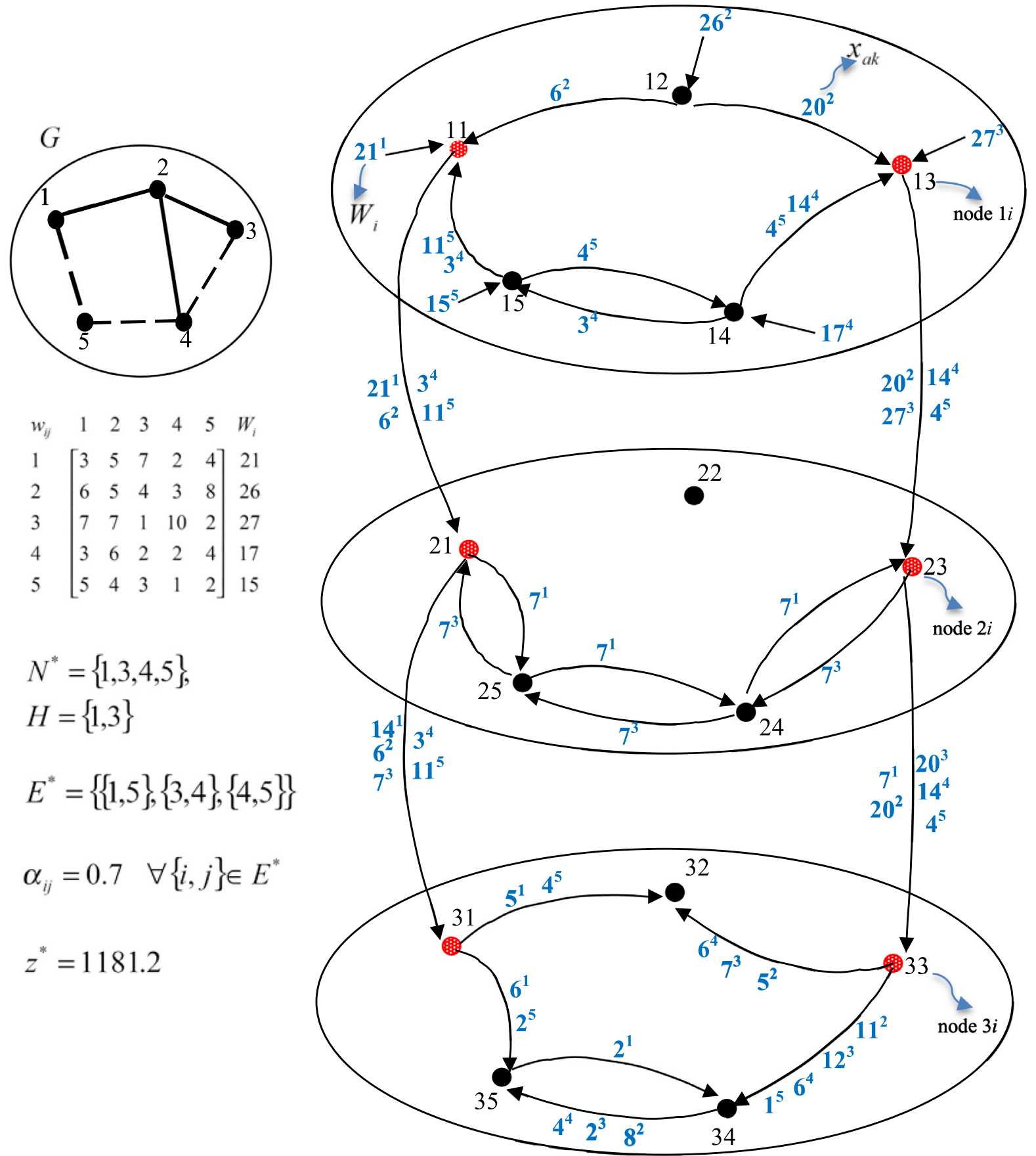

$$
\begin{aligned}
& N^{*}=\{1,3,4,5\}, \\
& H=\{1,3\} \\
& E^{*}=\{\{1,5\},\{3,4\},\{4,5\}\} \\
& \alpha_{i j}=0.7 \quad \forall\{i, j\} \in E^{*} \\
& z^{*}=1181.2
\end{aligned}
$$

Fig. 7. Optimal solution for the hub-arc location problem with $r=3$ and $v=1$.

by defining edges only between adjacent cities and assigning direct distances between them as the lengths. TR81 network is preprocessed via Floyd's algorithm (Floyd, 1962) to obtain complete versions of the TR81 problems, say TR81C. The results for RMCFP (CMCFP) are obtained by using TR81 (TR81C) instances. Note that TR81 data set is different from TR data set used in the hub location literature, e.g., (Alumur et al., 2009; Calik et al., 2009; Beasley, 1990). TR81 data set is defined on the non-complete network while TR data set is defined on the complete network.

There are no hub location models in the literature that address G-UMApHMP and hence that are directly comparable to MCFP with respect to solvability. However, to give an idea about the solvability of the proposed models, we select two models that address UMApHMP, a special case of G-UMApHMP, for comparison. One is the model EK of Ernst and Krishnamoorthy (1998) and the other is the model MCL of Marin et al. (2006). We will discuss these models in the next section. Both models are based on the complete-MN network structure. Accordingly, the results for these models are obtained by using TR81C.

PMED problems are defined on the non-complete PMED networks (RealN) and generally used for the $p$-median problems (Beasley, 1990). We use the networks of instances 6, 11, 16, and 21 with the number of nodes changing from 200 to 500 to define test problems. PMED problems are used to test the performances of RMCFP and the heuristic for large-scale problems. These instances are not possible to solve with CMCFP, EK, and MCL due to memory problems. There are famous data sets, e.g., CAB and AP (Beasley, 1990), in the hub location literature. However, these data 


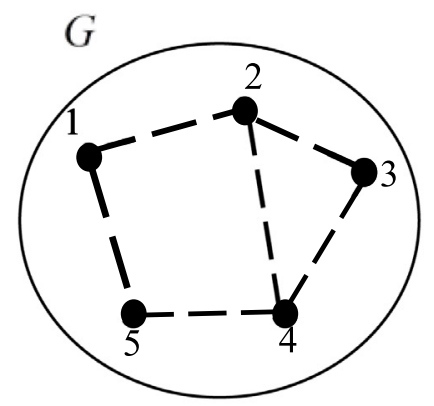

$$
\begin{aligned}
& \begin{array}{lllllll}
w_{i j} & 1 & 2 & 3 & 4 & 5 & W_{i}
\end{array} \\
& 1 \quad\left[\begin{array}{lllll}
3 & 5 & 7 & 2 & 4
\end{array}\right] 21 \\
& \begin{array}{llllll|l}
2 & 6 & 5 & 4 & 3 & 8 & 26
\end{array} \\
& \begin{array}{lllllll|l}
3 & 7 & 7 & 1 & 10 & 2 & 27
\end{array} \\
& \begin{array}{l|lllll|l}
4 & 3 & 6 & 2 & 2 & 4 & 17 \\
5 & 5 & 4 & 3 & 1 & 2 & 15
\end{array}
\end{aligned}
$$$$
N^{*}=H=\{1,2,3,4,5\}
$$$$
E^{*}=\{\{1,2\},\{1,5\},\{2,3\},
$$

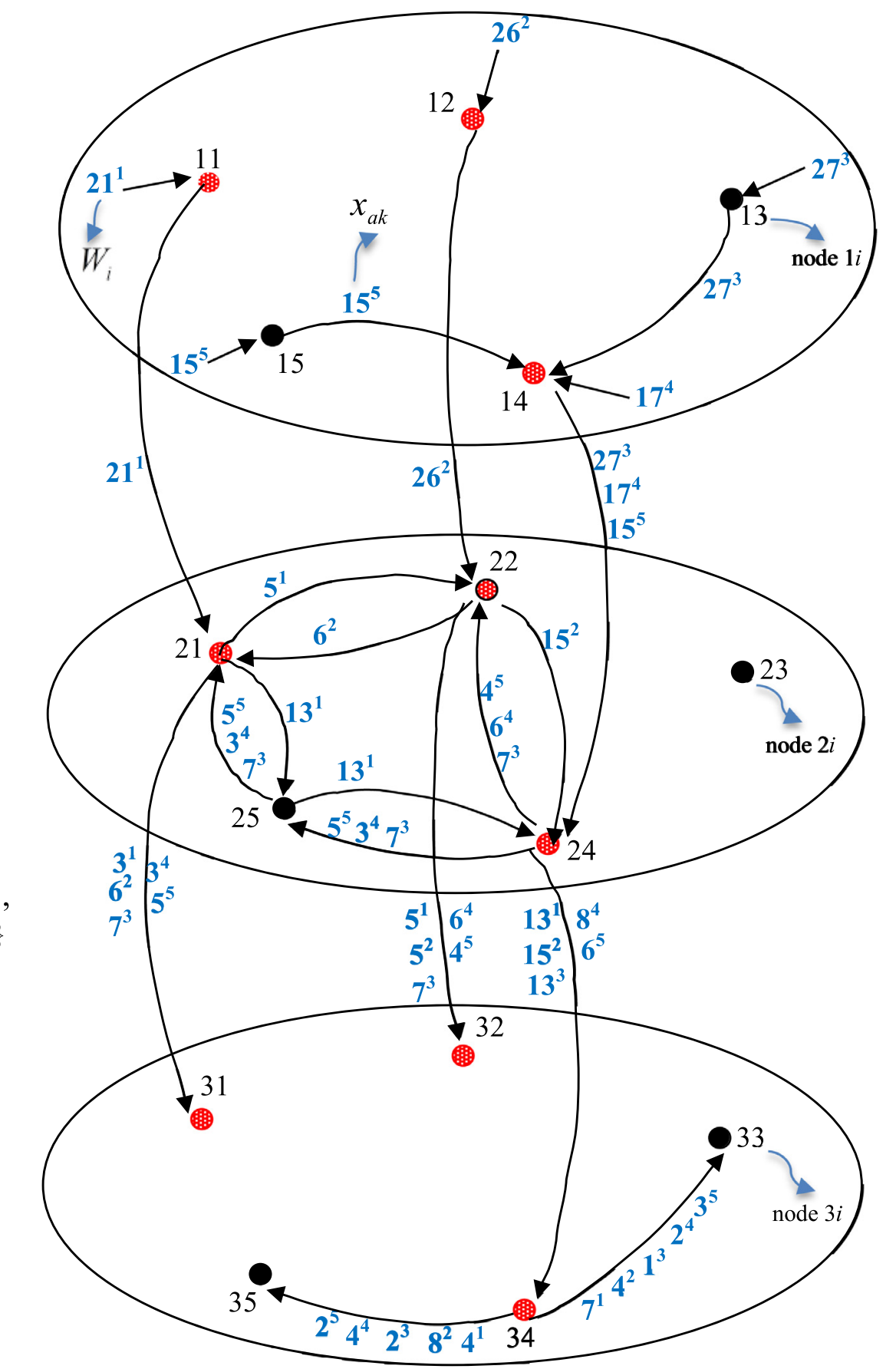

$$
\begin{aligned}
& \{2,4\},\{3,4\},\{4,5\}\} \\
\alpha_{i j}= & 0.7 \quad \forall\{i, j\} \in E^{*} \\
z^{*}= & 972.4
\end{aligned}
$$

Fig. 8. Optimal solution for the single-assignment case.

sets are defined on complete networks. In this regard, we use the PMED instances defined on non-complete networks.

Different problem instances are constructed by changing $H$ and $p$ where $E^{*}=E$ and $N^{*}=N$. $H \subset N^{*}$ for all instances except when $|H|=|N| . \quad \chi_{i j}=\delta_{i j}=1$, and $\alpha_{i j}=0.7 . w_{i j}$ is assigned randomly from a uniform distribution with the interval $(10,30)$. The characteristics of all test problems are given in Table 1.

Tests are performed on a PC with $2.50 \mathrm{GHz}$ Intel Core i7-4710HQ processor and 16 GB of RAM by using the solver CPLEX 12.1 (IBM ILOG (IBM ILOG, 2009)). The models are run until the optimality is attained or for $18,000 \mathrm{~s}$ at maximum.

We test all models under two different settings of CPLEX parameters. The first setting S1 uses default values and allows cut generation while the second setting S2 uses default values but does not allow cut generation. In both settings, file storage is set to 3 , which allows tree file to be stored on the hard disk when it reaches the default limit in order not to run out of memory (IBM ILOG, 2009).

Test results indicate that the performances of RMCFP and CMCFP change significantly for some instances under S1 and S2 although RMCFP performs better than CMCFP for all instances under both settings. However, the performances of EK and MCL are essentially the same under S1 and S2. That is, using S1 and S2 makes a difference only for the proposed models. In this regard, we report the results of the setting under which RMCFP performs better (with the smallest gap in the allotted time or the solution time 
Table 1

Problem characteristics.

\begin{tabular}{|c|c|c|c|c|c|c|c|c|c|c|}
\hline Pr.Id. & Instance type & $|N|$ & $|H|$ & $|D|$ & $|S|$ & $p$ & $\chi_{i j}$ & $\delta_{i j}$ & $\alpha_{i j}$ & $w_{i j}$ \\
\hline 1 & TR81(C) & 81 & 30 & 81 & 81 & 2 & 1 & 1 & 0.7 & Uniform $(10,30)$ \\
\hline 2 & TR81(C) & 81 & 30 & 81 & 81 & 3 & 1 & 1 & 0.7 & Uniform $(10,30)$ \\
\hline 3 & TR81(C) & 81 & 30 & 81 & 81 & 5 & 1 & 1 & 0.7 & Uniform $(10,30)$ \\
\hline 4 & TR81(C) & 81 & 50 & 81 & 81 & 2 & 1 & 1 & 0.7 & Uniform $(10,30)$ \\
\hline 5 & TR81(C) & 81 & 50 & 81 & 81 & 3 & 1 & 1 & 0.7 & Uniform $(10,30)$ \\
\hline 6 & TR81(C) & 81 & 50 & 81 & 81 & 5 & 1 & 1 & 0.7 & Uniform $(10,30)$ \\
\hline 7 & TR81(C) & 81 & 50 & 81 & 81 & 8 & 1 & 1 & 0.7 & Uniform $(10,30)$ \\
\hline 8 & TR81(C) & 81 & 50 & 81 & 81 & 10 & 1 & 1 & 0.7 & Uniform $(10,30)$ \\
\hline 9 & TR81(C) & 81 & 60 & 81 & 81 & 2 & 1 & 1 & 0.7 & Uniform $(10,30)$ \\
\hline 10 & TR81(C) & 81 & 60 & 81 & 81 & 3 & 1 & 1 & 0.7 & Uniform $(10,30)$ \\
\hline 11 & TR81(C) & 81 & 60 & 81 & 81 & 5 & 1 & 1 & 0.7 & Uniform $(10,30)$ \\
\hline 12 & TR81(C) & 81 & 60 & 81 & 81 & 8 & 1 & 1 & 0.7 & Uniform $(10,30)$ \\
\hline 13 & TR81(C) & 81 & 60 & 81 & 81 & 10 & 1 & 1 & 0.7 & Uniform $(10,30)$ \\
\hline 14 & TR81(C) & 81 & 81 & 81 & 81 & 2 & 1 & 1 & 0.7 & Uniform $(10,30)$ \\
\hline 15 & TR81(C) & 81 & 81 & 81 & 81 & 3 & 1 & 1 & 0.7 & Uniform $(10,30)$ \\
\hline 16 & TR81(C) & 81 & 81 & 81 & 81 & 5 & 1 & 1 & 0.7 & Uniform $(10,30)$ \\
\hline 17 & TR81(C) & 81 & 81 & 81 & 81 & 8 & 1 & 1 & 0.7 & Uniform $(10,30)$ \\
\hline 18 & TR81(C) & 81 & 81 & 81 & 81 & 10 & 1 & 1 & 0.7 & Uniform $(10,30)$ \\
\hline 19 & PMED6 & 200 & 200 & 200 & 200 & 2 & 1 & 1 & 0.7 & Uniform $(10,30)$ \\
\hline 20 & PMED6 & 200 & 200 & 200 & 200 & 5 & 1 & 1 & 0.7 & Uniform $(10,30)$ \\
\hline 21 & PMED6 & 200 & 200 & 200 & 200 & 8 & 1 & 1 & 0.7 & Uniform $(10,30)$ \\
\hline 22 & PMED6 & 200 & 200 & 200 & 200 & 10 & 1 & 1 & 0.7 & Uniform $(10,30)$ \\
\hline 23 & PMED11 & 300 & 300 & 300 & 300 & 2 & 1 & 1 & 0.7 & Uniform $(10,30)$ \\
\hline 24 & PMED11 & 300 & 300 & 300 & 300 & 5 & 1 & 1 & 0.7 & Uniform $(10,30)$ \\
\hline 25 & PMED11 & 300 & 300 & 300 & 300 & 8 & 1 & 1 & 0.7 & Uniform $(10,30)$ \\
\hline 26 & PMED11 & 300 & 300 & 300 & 300 & 10 & 1 & 1 & 0.7 & Uniform $(10,30)$ \\
\hline 27 & PMED16 & 400 & 400 & 400 & 400 & 2 & 1 & 1 & 0.7 & Uniform $(10,30)$ \\
\hline 28 & PMED16 & 400 & 400 & 400 & 400 & 5 & 1 & 1 & 0.7 & Uniform $(10,30)$ \\
\hline 29 & PMED16 & 400 & 400 & 400 & 400 & 8 & 1 & 1 & 0.7 & Uniform $(10,30)$ \\
\hline 30 & PMED16 & 400 & 400 & 400 & 400 & 10 & 1 & 1 & 0.7 & Uniform $(10,30)$ \\
\hline 31 & PMED21 & 500 & 500 & 500 & 500 & 2 & 1 & 1 & 0.7 & Uniform $(10,30)$ \\
\hline 32 & PMED21 & 500 & 500 & 500 & 500 & 5 & 1 & 1 & 0.7 & Uniform $(10,30)$ \\
\hline 33 & PMED21 & 500 & 500 & 500 & 500 & 8 & 1 & 1 & 0.7 & Uniform $(10,30)$ \\
\hline 34 & PMED21 & 500 & 500 & 500 & 500 & 10 & 1 & 1 & 0.7 & Uniform $(10,30)$ \\
\hline
\end{tabular}

Table 2

Computational results for RMCFP (CMCFP) using TR81(C) instances. Bold values indicate the best BPs for each instance.

\begin{tabular}{|c|c|c|c|c|c|c|c|c|c|c|c|c|c|c|c|}
\hline \multirow[t]{2}{*}{ Pr. Id. } & \multirow[t]{2}{*}{$\mathrm{S} 1$ or S2 } & \multicolumn{7}{|l|}{ RMCFP } & \multicolumn{7}{|l|}{ CMCFP } \\
\hline & & $Z_{L P}^{*}$ & LPT & BP & LB & Gap (\%) & IPT & $T_{B P}^{F}$ & $z_{L P}^{*}$ & LPT & BP & LB & Gap (\%) & IPT & $T_{B P}^{F}$ \\
\hline 1 & $\mathrm{~S} 2$ & 95,056 & 4 & 126,682 & 126,682 & 0.0 & 301 & 45 & 95,056 & 3 & 126,682 & 126,682 & 0.00 & 197 & 110 \\
\hline 2 & $\mathrm{~S} 2$ & 91,665 & 3 & 114,076 & 114,076 & 0.0 & 1090 & 110 & 91,665 & 2 & 114,076 & 114,076 & 0.00 & 1475 & 140 \\
\hline 3 & $\mathrm{~S} 1$ & 88,162 & 2 & 103,003 & 103,003 & 0.0 & 1666 & 16 & 88,162 & 2 & 103,003 & 103,003 & 0.00 & 3393 & 320 \\
\hline 4 & $\mathrm{~S} 2$ & 91,522 & 9 & 125,785 & 125,785 & 0.0 & 3366 & 3250 & 91,522 & 10 & 125,785 & 125,785 & 0.00 & 4781 & 4620 \\
\hline 5 & S2 & 87,957 & 5 & 114,076 & 114,076 & 0.0 & 11,541 & 5800 & 87,957 & 11 & 114,076 & 91,832 & 8.05 & 18,000 & 2810 \\
\hline 6 & $\mathrm{~S} 1$ & 84,359 & 2 & 103,424 & 97,389 & 5.8 & 18,000 & 1050 & 84,359 & 9 & 103,432 & 94,151 & 8.97 & 18,000 & 8600 \\
\hline 7 & $\mathrm{~S} 1$ & 81,834 & 2 & 94,676 & 92,406 & 2.4 & 18,000 & 9500 & 81,834 & 5 & 94,973 & 89,880 & 5.36 & 18,000 & 13,200 \\
\hline 8 & $\mathrm{~S} 1$ & 80,859 & 1 & 91,289 & 90,104 & 1.3 & 18,000 & 3800 & 80,859 & 3 & 91,305 & 87,940 & 3.69 & 18,000 & 16,400 \\
\hline 9 & $\mathrm{~S} 2$ & 90,494 & 7 & 125,785 & 125,785 & 0.0 & 3826 & 350 & 90,494 & 19 & 125,785 & 125,785 & 0.00 & 11,953 & 8350 \\
\hline 10 & $\mathrm{~S} 2$ & 86,893 & 5 & 114,076 & 114,076 & 0.0 & 4900 & 3800 & 86,893 & 16 & 114,363 & 93,573 & 18.2 & 18,000 & 2900 \\
\hline 11 & $\mathrm{~S} 1$ & 83,242 & 5 & 102,765 & 96,155 & 6.4 & 18,000 & 1890 & 83,242 & 21 & 103,399 & 93,193 & 9.87 & 18,000 & 7540 \\
\hline 12 & $\mathrm{~S} 1$ & 80,698 & 3 & 94,061 & 91,306 & 2.9 & 18,000 & 4300 & 80,698 & 19 & 94,113 & 89,009 & 5.42 & 18,000 & 16,800 \\
\hline 13 & $\mathrm{~S} 1$ & 79,709 & 3 & 91,093 & 88,923 & 2.4 & 18,000 & 10,200 & 79,709 & 9 & 91,404 & 87,153 & 4.65 & 18,000 & 3400 \\
\hline 14 & $\mathrm{~S} 2$ & 88,297 & 9 & 119,594 & 119,594 & 0.0 & 5009 & 780 & 88,297 & 43 & 119,594 & 100,535 & 15.9 & 18,000 & 2750 \\
\hline 15 & $\mathrm{~S} 1$ & 84,547 & 5 & 113,027 & 98,835 & 12.6 & 18,000 & 8300 & 84,547 & 35 & 114,076 & 96,686 & 15.2 & 18,000 & 7800 \\
\hline 16 & $\mathrm{~S} 1$ & 81,000 & 3 & 101,793 & 94,446 & 7.2 & 18,000 & 1300 & 81,000 & 29 & 102,235 & 92,141 & 9.87 & 18,000 & 4300 \\
\hline 17 & $\mathrm{~S} 1$ & 78,464 & 3 & 93,563 & 89,641 & 4.2 & 18,000 & 3700 & 78,464 & 19 & 95,385 & 88,324 & 7.4 & 18,000 & 16,100 \\
\hline 18 & $\mathrm{~S} 1$ & 77,500 & 2 & 90,572 & 87,502 & 3.4 & 18,000 & 4400 & 77,500 & 14 & 92,145 & 86,486 & 6.15 & 18,000 & 14,140 \\
\hline
\end{tabular}

if optimality is attained) for each problem. Accordingly, we report the results of EK and MCL under the same settings.

In the tables, we present linear programming (LP) relaxation bound $\left(z_{L P}^{*}\right)$, runtime in CPU seconds $(\mathrm{T})$, lower bound (LB) and the objective function value of the best integer solution at the end of runtime (BP), the time at which BP is achieved in the solution process $\left(T_{B P}^{F}\right)$, and relative optimality gap (Gap\%) for each instance. Objective values and bounds are expressed in thousands. The optimality gap in CPLEX is defined as $|B P-B F| /\left(10^{-10}+|B P|\right)$, where $\mathrm{BF}$ is the best remaining objective function value of any unexplored node (IBM ILOG, 2009).

\subsubsection{Assessing the performance of the proposed models using} optimization software

Table 2 gives computational results for TR81(C) instances. The results indicate that RMCFP performs better than CMCFP (with regard to the optimality gap achieved in the allotted time or the solution time if optimality is attained) under both S1 (with cuts) and S2 (without cuts) settings for all instances. However, we observe that the performances of the proposed models change significantly for some instances under S1 and S2 depending on the problem characteristics. Specifically, for problems with $p=2,3(p \geq 5)$, the models generally perform better under S2 (S1). When S1 is used, in 
Table 3

Comparison of heuristic results to best solutions for TR81 and PMED instances. $B P^{\text {best }}$ column indicates which models achieve the $B P^{\text {best }}$ values.

\begin{tabular}{|c|c|c|c|c|c|c|c|}
\hline \multirow[t]{2}{*}{ Pr. Id. } & \multirow[t]{2}{*}{$B P^{\text {best }}$} & \multicolumn{2}{|c|}{$\operatorname{RFHEUR}(k=1)$} & \multicolumn{2}{|c|}{ RFHEUR $(k=2)$} & \multicolumn{2}{|c|}{ Gap_Heur (\%) } \\
\hline & & $B P^{\text {Heur }}$ & Time (s) & $B P^{\text {Heur }}$ & Time (s) & $k=1$ & $k=2$ \\
\hline 1 & 126,682 (All) & 128,131 & 9 & 126,682 & 6 & -1.14 & 0.00 \\
\hline 2 & 114,076 (All) & 116,037 & 4 & 116,037 & 7 & -1.72 & -1.72 \\
\hline 3 & $103,003($ All $)$ & 103,939 & 5 & 103,227 & 15 & -0.91 & -0.22 \\
\hline 4 & 125,785 (All) & 128,131 & 3 & 126,682 & 5 & -1.87 & -0.71 \\
\hline 5 & 114,076 (EK/RMCFP) & 115,161 & 3 & 115,161 & 7 & -0.95 & -0.95 \\
\hline 6 & $103,227(\mathrm{MCL})$ & 104,987 & 3 & 104,426 & 15 & -1.71 & -1.16 \\
\hline 7 & 94,676 (EK/RMCFP) & 96,521 & 3 & 95,647 & 48 & -1.95 & -1.03 \\
\hline 8 & 91,289(All) & 95,529 & 2 & 92,887 & 76 & -4.64 & -1.75 \\
\hline 9 & 125,785 (EK/RMCFP) & 128,131 & 3 & 126,682 & 4 & -1.87 & -0.71 \\
\hline 10 & 114,076 (EK/RMCFP) & 115,161 & 4 & 115,161 & 6 & -0.95 & -0.95 \\
\hline 11 & $102,235(\mathrm{MCL})$ & 104,678 & 10 & 104,101 & 16 & -2.39 & -1.82 \\
\hline 12 & 93,972(MCL) & 96,415 & 4 & 94,713 & 32 & -2.60 & -0.79 \\
\hline 13 & 91,093 (RMCFP) & 94,436 & 9 & 91,418 & 158 & -3.67 & -0.36 \\
\hline 14 & 119,594 (EK/RMCFP) & 119,941 & 3 & 119,941 & 3 & -0.29 & -0.29 \\
\hline 15 & $112,671(\mathrm{EK})$ & 115,837 & 4 & 115,488 & 8 & -2.81 & -2.50 \\
\hline 16 & 101,793 (RMCFP) & 104,460 & 4 & 103,800 & 8 & -2.85 & -2.20 \\
\hline 17 & 93,563 (RMCFP) & 96,118 & 5 & 94,580 & 15 & -2.73 & -1.09 \\
\hline \multirow[t]{4}{*}{18} & 90,572(RMCFP) & 93,797 & 3 & 93,389 & 22 & -3.56 & -3.11 \\
\hline & & & & & Max & -4.64 & -3.11 \\
\hline & & & & & Min & -0.29 & 0.00 \\
\hline & & & & & Average & -2.14 & -1.19 \\
\hline 19 & 94,759 & 88,918 & 45 & 87,893 & 65 & 6.16 & 7.25 \\
\hline 20 & 77,113 & 75,179 & 42 & 75,179 & 132 & 2.51 & 2.51 \\
\hline 21 & 70,943 & 71,298 & 49 & 70,979 & 814 & -0.50 & -0.05 \\
\hline 22 & 68,069 & 69,737 & 55 & 69,481 & 1872 & -2.45 & -2.08 \\
\hline 23 & 122,247 & 115,020 & 211 & 114,761 & 225 & 5.91 & 6.12 \\
\hline 24 & 110,511 & 106,471 & 227 & 106,047 & 583 & 3.66 & 4.04 \\
\hline 25 & 103,620 & 102,550 & 198 & 101,407 & 1276 & 1.03 & 2.14 \\
\hline 26 & 100,271 & 100,164 & 187 & 99,746 & 7213 & 0.11 & 0.52 \\
\hline 27 & 184,997 & 168,675 & 324 & 167,739 & 453 & 8.82 & 9.33 \\
\hline 28 & 166,819 & 151,111 & 539 & 150,574 & 1378 & 9.42 & 9.74 \\
\hline 29 & 145,281 & 143,609 & 576 & 143,292 & 2865 & 1.15 & 1.37 \\
\hline 30 & 152,048 & 140,914 & 563 & 139,512 & 894 & 7.32 & 8.25 \\
\hline 31 & 269,516 & 235,827 & 413 & 235,827 & 256 & 12.50 & 12.50 \\
\hline 32 & 213,830 & 212,151 & 376 & 212,087 & 1045 & 0.79 & 0.82 \\
\hline 33 & 209,625 & 204,833 & 403 & 204,267 & 4752 & 2.29 & 2.56 \\
\hline \multirow[t]{4}{*}{34} & 203,998 & 201,185 & 365 & 200,271 & 18,000 & 1.38 & 1.83 \\
\hline & & & & & Max & 12.50 & 12.50 \\
\hline & & & & & Min & -2.45 & -2.08 \\
\hline & & & & & Average & 3.76 & 4.18 \\
\hline
\end{tabular}

general, three types of cuts are generated: flow cuts (FCs), Gomory fractional cuts (GFCs), and mixed integer rounding cuts (MIRCs). FCs, GFCs, and MIRCs constitute on the average about 97, 0.3, and $2.7 \%$ of the total number of cuts generated, respectively. The number of FCs generated is almost the same as (half of) the number of original constraints for problems with $p \geq 5(p=2,3)$. Even though not sufficiently, the generated cuts help to increase LBs and decrease optimality gaps. However, for some instances with $p=2$, 3 , GFCs affect the solution of the models adversely. The effect of S1 and S2 is the same for both RMCFP and CMCFP. That is, if one is affected favorably (adversely), the other one is affected favorably (adversely) as well. In this regard, in Table 2, we present the results of the setting under which RMCFP performs better and discuss the results for RMCFP.

LP relaxations of RMCFP are solved in a matter of seconds. However, the LP bounds are weak; the gaps between $z_{L P}^{*}$ and BP change from about 15 to $25 \%$. In this regard, RMCFP spends most of the runtime to increase LBs. RMCFP optimally solves 8 problems out of 18 in the allotted time. For problems not solved to optimality, the resulting gaps between BPs and LBs change from 1.3 to 12.6\% (Gap column). However, when the gaps are computed using the best LBs (obtained by solving EK and MCL and given in Table 4) instead of the ones in Table 2, the gaps turn out to change from 0.42 to $2.15 \%$ and the highest gap of $12.6 \%$ for Pr.15 decreases to $1.31 \%$. Considering this result and that BPs in Table 2 are attained within about $3600 \mathrm{~s}$ except for a few instances $\left(T_{B P}^{F}\right.$ column under RMCFP), we conclude that RMCFP can find near-optimal solutions in a reasonable amount of time.

We think that good solutions are obtained with RMCFP in a short time due to two reasons: (1) The model is actually a network flow model with side constraints and it is known that network flow models are easier to solve. When cuts are added, this structure may not be preserved. In this regard, better BPs are generally obtained when cuts are not allowed. (2) The model size is considerably small in comparison to other models. For TR81(C) instances, the number of variables in RMCFP is between 70-90\% less than that in CMCFP, EK, and MCL and the number of constraints in RMCFP and CMCFP is between 90-95\% less than that in EK and MCL. Note that CMCFP, the complete-MN network version of MCFP, has also considerably less number of constraints than the corresponding models EK and MCL.

\subsubsection{Assessing the performance of the heuristic RFHEUR}

We assess the performance of RFHEUR by comparing $B P^{\text {best }}$ (the best BP value attained by any of the models RMCFP, CMCFP, EK, or $\mathrm{MCL}$ ) and $B P^{\text {Heur }}$ (the objective function value found by RFHEUR) for TR81 and PMED instances. For TR81 instances, $B P^{\text {best }}$ values are equivalent to the smallest of the BP values in Tables 2 and 4. For PMED instances, $B P^{b e s t}$ values are obtained by running RMCFP for $18,000 \mathrm{~s}$ under S2 becuase solutions with better BP values are gen- 
Table 4

Computational results for EK and MCL using TR81C instances. Bold (Italic) values indicate the best BPs (LBs) for each instance.

\begin{tabular}{|c|c|c|c|c|c|c|c|c|c|c|c|c|c|}
\hline \multirow[t]{2}{*}{ Pr. Id. } & \multirow[t]{2}{*}{$\mathrm{S} 1$ or S2 } & \multicolumn{6}{|l|}{ EK } & \multicolumn{6}{|l|}{ MCL } \\
\hline & & $z_{L P}^{*}$ & LPT & $\mathrm{BP}$ & LB & Gap (\%) & IPT & $z_{L P}^{*}$ & LPT & $\mathrm{BP}$ & LB & Gap (\%) & IPT \\
\hline 1 & $\mathrm{~S} 2$ & 126,201 & 812 & 126,682 & 126,682 & 0.00 & 1203 & 126,201 & 2058 & 126,682 & 126,682 & 0.00 & 2862 \\
\hline 2 & $\mathrm{~S} 2$ & 113,163 & 567 & 114,076 & 114,076 & 0.00 & 835 & 113,163 & 769 & 114,076 & 114,076 & 0.00 & 1560 \\
\hline 3 & $\mathrm{~S} 1$ & 101,653 & 439 & 103,003 & 103,003 & 0.00 & 1178 & 101,653 & 684 & 103,003 & 103,003 & 0.00 & 2141 \\
\hline 4 & $\mathrm{~S} 2$ & 125,201 & 2514 & 125,785 & 125,785 & 0.00 & 3909 & 125,201 & 9427 & 125,785 & 125,785 & 0.00 & 12,652 \\
\hline 5 & $\mathrm{~S} 2$ & 112,586 & 1885 & 114,076 & 114,076 & 0.00 & 5783 & 112,586 & 7023 & 114,146 & 113,505 & 0.56 & 18,000 \\
\hline 6 & $\mathrm{~S} 1$ & 100,624 & 2177 & 103,352 & 102,993 & 0.35 & 18,000 & 100,624 & 5454 & 103,226 & 101,809 & 0.00 & 18,000 \\
\hline 7 & $\mathrm{~S} 1$ & 92,869 & 1964 & 94,676 & 93,890 & 0.83 & 18,000 & 92,869 & 3080 & 95,034 & 93,396 & 1.72 & 18,000 \\
\hline 8 & $\mathrm{~S} 1$ & 89,899 & 1728 & 91,289 & 90,670 & 0.67 & 18,000 & 89,899 & 2009 & 91,289 & 90,538 & 0.82 & 18,000 \\
\hline 9 & $\mathrm{~S} 2$ & 125,200 & 4197 & 125,785 & 125,785 & 0.00 & 5502 & & & No Soluti & & & \\
\hline 10 & $\mathrm{~S} 2$ & 112,364 & 2829 & 114,076 & 114,076 & 0.00 & 11,075 & & & No Soluti & & & \\
\hline 11 & $\mathrm{~S} 1$ & 100,167 & 3230 & 102,476 & 100,984 & 1.47 & 18,000 & 100,167 & 5829 & 102,235 & 100,933 & 1.27 & 18,000 \\
\hline 12 & $\mathrm{~S} 1$ & 92,502 & 2610 & 94,061 & 93,132 & 0.99 & 18,000 & 92,502 & 4387 & 939,721 & 930,897 & 0.94 & 18,000 \\
\hline 13 & $\mathrm{~S} 1$ & 89,591 & 2731 & 91,149 & 90,030 & 1.23 & 18,000 & 89,591 & 4218 & 91,404 & 90,033 & 1.50 & 18,000 \\
\hline 14 & $\mathrm{~S} 2$ & 119,575 & 4950 & 119,594 & 119,594 & 0.00 & 4869 & & & No Soluti & & & \\
\hline 15 & $\mathrm{~S} 1$ & 110,885 & 8338 & 112,671 & 111,548 & 0.99 & 18,000 & & & No Soluti & & & \\
\hline 16 & $\mathrm{~S} 1$ & 99,335 & 5537 & 101,956 & 99,602 & 2.31 & 18,000 & 99,335 & 14,705 & 107,111 & 99,453 & 7.15 & 18,000 \\
\hline 17 & $\mathrm{~S} 1$ & 91,733 & 4852 & 93,632 & 91,915 & 1.83 & 18,000 & 91,733 & 11,407 & 94,900 & 91,884 & 3.18 & 18,000 \\
\hline 18 & $\mathrm{~S} 1$ & 88,983 & 4752 & 90,756 & 89,177 & 1.74 & 18,000 & 88,983 & 13,540 & 92,531 & 89,106 & 3.70 & 18,000 \\
\hline
\end{tabular}

erally obtained with S2. RMCFP can find feasible solutions for all PMED problems but cannot solve any problems to optimality. The resulting optimality gaps change from 20 to $37 \%$ but when S1 is used, the resulting gaps decrease by about $10 \%$.

We implement RFHEUR for $k=1,2$ under S2 with the maximum allowable time of $18,000 \mathrm{~s}$. Table 3 gives the results for $k=1$, 2. The solution times of RFHEUR for TR81 and PMED instances are negligible for $k=1$. This is expected because RMCFP_Rel is easy to solve and RMCFP in the second step is solved as an LP because the location decisions are fixed. For $k=2$, the solution times for TR81 are higher than those obtained for $k=1$ but still negligible. For PMED instances, solution times increase considerably but still acceptable when the sizes of the problems are considered. High solution times result because solving RMCFP to select $p$ locations out of $2 p$ candidates becomes difficult for large values of $p$ and largescale networks. Specifically, for Pr.Id. 34, the optimal solution for RMCFP cannot be found in the allotted time. This is an example of a situation where the restricted RMCFP may be as difficult to solve as the original problem.

The last two columns (Gap_Heur) in Table 3 give the gaps between $B P^{\text {best }}$ and $B P^{\text {Heur }}$ and are defined as $100 \times\left(B P^{\text {best }}-B P^{x}\right) / B P^{\text {best }}$. Negative (positive) values indicate that $B P^{\text {best }}\left(B P^{\text {Heur }}\right)$ is better than $B P^{\text {Heur }}$ (BPbest). For TR81 problems, $B P^{\text {best }}$ values are better than or equal to $B P^{\text {Heur }}$. However, the worst (average) gaps are $4.64 \%$ (2.14\%) and $3.11 \%$ (1.19) for $k=1$ and $k=2$, respectively. For PMED problems, $B P^{\text {best }}$ values are better than or equal to $B P$ Heur for only two instances. For all other instances, RFHEUR attains better objective function values. The improvement over $B P^{\text {best }}$ is on the average about 3.76 and $4.18 \%$ for $k=1$ and $k=2$, respectively, but increases up to $12.5 \%$ for one instance. Considering that RMCFP can find good solutions in a short time and that RFHEUR can improve upon $B P^{\text {best }}$ values, we think that RFHEUR can be very useful especially for large-scale problems.

\section{Comparison of the proposed models with the previous models}

Even though not directly comparable to MCFP, EK and MCL are chosen for comparison because (1) MCL is closer to MCFP in the sense that it can handle complete networks whose costs do not satisfy the triangle inequality (even though other classical assumptions do hold) and (2) EK is known to have the best performance with standard software (e.g., Alumur and Kara, 2008). The models
EK and MCL are given in Appendix B for reference. Table 4 gives computational results for EK and MCL for TR81C instances. Note that we present the results of the same settings (S1 or S2) in Table 2.

We know that there are better ways of solving EK and MCL. However, our purpose is just to give an idea about the performance of the proposed models. In this respect, we will state main findings: (1) The performances of EK and MCL are essentially the same under S1 and S2. We think that this results from the fact that a small number of cuts is generated when S1 is used because the LP bounds are much better in comparison to those of the proposed models. (The gaps between $z_{L P}^{*}$ and BPs change from 0.02 to 2.63\%.) (2) EK performs much better than MCL. MCL is obtained by adding some constraints to the model EK to develop a model that can be used when the triangle inequality is not satisfied. This indicates that it may be challenging not only modeling-wise but also computationally to incorporate several features of MCFP into the current models. (3) The performances of RMCFP and EK are comparable. The resulting optimality gaps for EK are better than those of RMCFP due to the weak LP bounds of RMCFP. However, RMCFP performs similar to and sometimes better than EK with respect to the BP values attained. Moreover, RMCFP finds good BP values in much shorter times as discussed above. (4) The solution times of LP relaxations of the proposed models are much better than those of the previous models; which can be utilized in developing advanced solution algorithms for the previous or to-be-developed models.

\section{Conclusions}

We have introduced and studied the Generalized Uncapacitated Multiple-Allocation p-Hub Median Problem (G-UMApHMP) for noncomplete networks. Our main contribution is to develop a problem setting and modeling approach that allow several basic assumptions about hub location problems to be relaxed and hence add flexibility and realism in modeling several characteristics of reallife hub networks. The proposed models work correctly with any type of underlying network (complete or not) and cost structure (satisfying the triangle inequality or not). We have also proposed a heuristic based on the proposed modeling aproach. Computational studies with medium- and large-scale problems indicate that the models can find good solutions in a short time and the heuristic is especially effective for large-size problems.

Further research is needed to develop better solution algorithms that use the advantages of the proposed modeling approach 
to find exact solutions for large-size problems. There also occurs a need to increase the LP bounds without worsening the solvability of the models. New models for different hub location problems may also be developed by using the proposed modeling approach.

\section{Acknowledgments}

This research was supported by the Scientific and Technological Research Council of Turkey (TÜBiTAK Grant No: 114M363). The authors are grateful to anonymous referees for providing constructive feedback that has helped improve in major ways the presentation of the material in the paper.

\section{Appendix A. Sizes of the models MCFP and RMCFP}

Let $n=|N|$ and $n^{*}=\left|N^{*}\right|$. The number of real variables is $\left(4|E|+2\left|E^{*}\right|+2|H|\right)|S|$ with the order of at most $n^{3}$ while the number of binary variables is $|H|$ with the order of at most $n$ in MCFP. In RMCFP, the number of variables $x_{a k}$ is reduced from $\left(4|E|+2\left|E^{*}\right|+2|H|\right)|S|$ to $[(n-1)+|H||H-1|+(n-1)|H|+2|H|]|S|$. When the network is planar as in most real-life networks, there are significant savings in the number of flow variables. The number of real variables for planar networks is at most $(20 n-36) n$ with the order of at most $n^{2}$ while it is $3 n^{3}-n^{2}$ in complete networks with the order of at most $n^{3}$. The number of flow-balance constraints is $\left(2 n+n^{*}\right)|S|$ with the order of at most $3 n^{2}$ and the number of flow-passage constraints is $2|H||S|$ with the order of at most $2 n^{2}$.

\section{Appendix B. Previous models}

\section{B.1. The model of Ernst and Krishnamoorthy (1998)}

Consider a set of $n$ locations (points), each of which is producing some product and sending it to the others. Let $w_{i j}$ be the amount of product to be sent from point $i$ to point $j$ and $d_{i j}$ be the transportation cost from point $i$ to point $j$ for all $i, j=1, \ldots, n$. A set of $p$ points is to be chosen as hubs.

Let $O_{i}=\sum_{j} w_{i j}, i=1, \ldots, n$, be the units of product which must be sent from origin $i$ and $D_{j}=\sum_{i} w_{i j}, j=1, \ldots, n$, be the units of product that must be sent to destination $j$. For all $i, j, k, m=1, \ldots, n$, the decision variables in the formulation are (1) $Y_{k}$,which is 1 if $k$ is a hub and 0 otherwise, (2) $Z Z_{i k}$, which is the amount of product going from origin $i$ directly to hub $k$, (3) $H_{i k m}$, which is the amount of product going from origin $i$ directly to hub $k$ and then directly to hub $m$, and (4) $N N_{i j m}$, which is the amount of product going from origin $i$ to any hub and then to hub $m$ and finally to destination $j$. $\forall i, j$

Model EK: The formulation of Ernst and Krishnamoorthy (1998)

$$
\begin{aligned}
& z^{*}=\min _{Y, N N, H, Z Z} \sum_{i, k} d_{i k} Z Z_{i k}+\sum_{i, k, m} \alpha d_{k m} H_{i k m}+\sum_{i, j, m} d_{m j} N N_{i j m} \\
& \text { s.t. } \quad \sum_{m} N N_{i j m}=w_{i j} \quad \forall i, j \\
& \sum_{m} H_{i l m}+\sum_{j} N N_{i j l}-Z Z_{i l}-\sum_{k} H_{i k l}=0 \quad \forall i, l \\
& Z Z_{i k} \leq O_{i} Y_{k} \quad \forall i, j \\
& \sum_{i} N N_{i j m} \leq D_{j} Y_{m} \quad \forall j, m \\
& \sum_{k} Y_{k}=p
\end{aligned}
$$

$$
\begin{array}{cl}
Y_{k} \in\{0,1\} & \forall k \\
N N_{i j m}, H_{i k m}, Z Z_{i k} \geq 0 & \forall i, j, k, m
\end{array}
$$

Objective function (18) minimizes the total cost. Constraints (19) are demand-satisfaction constraints. Constraints (20) are flow balance constraints associated with the intermediate points between origin-destination pairs. Constraints (21) and (22) ensure that there is no flow traversing an intermediate node which is not a hub. Constraints (23) set the number of hubs to be located. Constraints (24) and (25) define the decision variables.

\section{B.2. The model of Marin et al. (2006)}

Model EK assumes that the distances satisfy the triangle inequality. Otherwise, the model fails because optimal paths connecting two nodes may include more than two hubs and optimal paths can traverse non-hub nodes. To get over this difficulty, Marin et al. (2006) propose adding a set of constraints to EK, which is given next.

Model MCL: The formulation of Marin et al. (2006)

In addition to (18), (20)-(25);

$$
\begin{array}{cc}
\sum_{m} H_{i k m} \leq O_{i} Y_{k} & \forall i, k \\
\sum_{m} H_{i k m} \leq O_{i} Y_{m} & \forall i, m \\
\sum_{m} H_{i k m} \leq Z Z_{i k} & \forall i, k \\
Z Z_{i i} \geq O_{i} Y_{i} \quad \forall i & \\
N N_{i j j} \geq w_{i j} Y_{j} \quad \forall i, j &
\end{array}
$$

Constraints (26) and (27) force the flow represented by the variables $H$ to go from one hub to another. Constraints (29) imply that when the origin is a hub, there is an initial flow inside the same node. Constraints (30) imply that when the destination is a hub, there is a final flow inside the same node. Constraints (28) limit the number of points traversed by a flow, using the flow which directly comes from the origin as a bound on the outcoming flow, represented by variables $H$.

\section{References}

Alumur, S., Kara, B.Y., 2008. Network hub location problems: the state of the art. Eur. J. Oper. Res. 190, 1-21.

Alumur, S., Kara, B.Y., Karasan, O.E., 2009. The design of single allocation incomplete hub networks. Transp. Res. part B 43, 936-951.

Beasley, J.E., 1990. OR-Library: distributing test problems by electronic mail. J. Oper. Res. Soc. 41 (11), 1069-1072.

Calik, H., Alumur, S., Kara, B.Y., Karasan, O.E., 2009. A tabu-search based heuristic for the hub covering problem over incomplete hub networks. Comput. Oper. Res. 36, 3088-3096.

Camargo, R.S., Miranda, G., Luna, H.P., 2008. Benders decomposition for the uncapacitated multiple allocation hub location problem. Comput. Oper. Res. 35 (4), 1047-1064.

Camargo, R.S., Miranda, G., Luna, H.P., 2009. Benders decomposition for hub location problems with economies of scale. Transp. Sci. 43, 86-97.

Campbell, J.F., O'Kelly, M.E., 2012. Twenty-five years of hub location research. Trans. Sci. 46, 153-169.

Campbell, J.F., Ernst, A.T., Krishnamoorthty, M., 2001. Hub Location Problems. In: Hamacher, H., Drezner, Z. (Eds.), Location Theory: Applications and Theory. Springer-Verlag, pp. 373-406.

Campbell, J.F., Ernst, A., Krishnamoorthy, M., 2005a. Hub arc location problems: part i - introduction and results. Manag. Sci. 51, 1540-1555.

Campbell, J.F., Ernst, A., Krishnamoorthy, M., 2005b. Hub arc location problems: part II - formulations and optimal algorithms. Manag. Sci. 51, 1556-1571. 
Campbell, J.F., De Miranda, G., De Camargo, R.S., O’Kelly, M.E., 2015. Hub location and network design with fixed and variable costs. In: Proceedings of the Forty Eighth Annual Annual Hawaii International Conference on System Sciences (HICSS), pp. 1059-1067.

Campbell, J.F., 1992. Location and allocation for distribution systems with transshipments and transportation economies of scale. Ann. Oper. Res. 40, 77-99.

Campbell, J.F., 2010. Designing hub networks with connected and isolated hubs. HICSS Proc. 1-10.

Campbell, J.F., 2013. Modeling economies of scale in transportation hub networks. In: Proceedings of the Forty Sixth Annual Annual Hawaii International Conference on System Sciences (HICSS), pp. 1154-1163.

Contreras, I., Fernández, E., Marín, A., 2010. The tree of hubs location problem. Eur. J. Oper. Res. 202 (2), 390-400.

Contreras, I., Cordeau, J.-F., Laporte, G., 2011. Benders decomposition for large-scale uncapacitated hub location. Oper. Res. 59 (6), 1477-1490.

Contreras, I., 2015. Hub location problems. In: Laporte, G., Nickel, S., Saldanha da Gama, F (Eds.), Location Science. Springer, pp. 311-344.

Ernst, A.T., Krishnamoorthy, M., 1998. Exact and heuristic algorithms for the uncapacitated multiple allocation p-hub median problem. Eur. J. Oper. Res. 104, $100-112$.

Floyd, R.W., 1962. Algorithm 97: shortest path. Commun. Assoc. Comput. Mach. 5 (6), 345.
Marin, A., Canovas, L., Landete, M, 2006. New formulations for the uncapacitated multiple allocation hub location problem. Eur. J. Oper. Res. 172 (1), 274-292.

Martins de Sá, E. de Camargo, R.S., de Miranda, R, 2013. An improved benders decomposition algorithm for the tree of hubs location problem. Eur. J. Oper. Res. 226, 185-202.

Martins de Sá, E., Contreras, I., Cordeau, J., 2015a. Exact and heuristic algorithms for the design of hub networks with multiple lines. Eur. J. Oper. Res. 246, 186-198.

Martins de Sá, E., Contreras, I., Cordeau, J.-F., Saraiva de Camargo, R., de Miranda, G., 2015b. The hub line location problem. Transp. Sci. 49 (3), 500-518.

Nishizeki, T., Chiba, N., 1998. Planar Graphs: Theory And Algorithms, Annals of Discrete Mathematics. Elsevier, p. 32.

O'Kelly, M.E., Bryan, D, 1998. Hub location with flow economies of scale. Transp. Res. part B 32, 605-616.

IBM ILOG, IBM ILOG CPLEX V12.1, User's Manual for CPLEX, 2009.

Ricardo-AEA. Update of the handbook on external costs of transport. final report to european commission, Report Number ED57769, UK, 2014.

Skorin-Kapov, D., Skorin-Kapov, J., O’Kelly, M, 1996. Tight linear programming relaxations of uncapacitated p-hub median problems. Eur. J. Oper. Res. 94, 582-593.

Transportation Research Board. National cooperative freight research program report 22: cost data elements. transportation research board of the national academies, Washingon DC., 2013.

Yaman, H, 2009. The hierarchical hub median problem with single assignment. Transp. Res. part B 43, 643-658. 